\title{
On predicting receptivity in a compressible infinite swept wing boundary layer
}

\author{
Christian Thomas, ${ }^{1}$, a) Shahid Mughal, ${ }^{1}$ and Richard Ashworth ${ }^{2}$ \\ ${ }^{1)}$ Department of Mathematics, South Kensington Campus, Imperial College London, London, SW7 $2 A Z$, \\ $U K$ \\ ${ }^{2)}$ Airbus Group Innovations, Bristol, BS99 7AR, UK
}

The receptivity of crossflow disturbances on an infinite swept wing is investigated using solutions of the adjoint linearised Navier-Stokes equations. The adjoint based method for predicting the magnitude of stationary disturbances generated by randomly distributed surface roughness is described, with the analysis extended to include both surface curvature and compressible flow effects. Receptivity is predicted for a broad spectrum of spanwise wavenumbers, variable freestream Reynolds numbers and subsonic Mach numbers. Curvature is found to play a significant role in the receptivity calculations, while compressible flow effects are only found to marginally affect the initial size of the crossflow instability. A Monte-Carlo type analysis is undertaken to establish the mean amplitude and variance of crossflow disturbances generated by the randomly distributed surface roughness. Mean amplitudes are determined for a range of flow parameters that are maximised for roughness distributions containing a broad spectrum of roughness wavelengths, including those that are most effective in generating stationary crossflow disturbances. A control mechanism is then developed where the short scale roughness wavelengths are damped, leading to significant reductions in the receptivity amplitude.

\section{INTRODUCTION}

Receptivity is the initial stage of the laminar-turbulent transition process, and describes how environmental features can excite fluctuations within the boundary layer. The mechanisms causing the generation of disturbances may be due to free-stream acoustic or vortical structures and (or coupled to) surface roughness ${ }^{1,2}$. Experimental observations demonstrate that transition is heavily dependent on the environmental characteristics ${ }^{3,4}$, which can also be influenced by three-dimensionality, surface curvature and the freestream conditions. Receptivity modelling establishes the initial magnitude of the fluctuations within the laminar boundary layer flow that essentially dictates the possible paths to transition of a laminar flow to turbulence. If the environmentally generated perturbations are sufficiently weak, the transition process is initially governed by linear disturbance development; which may be due to Tollmien-Schlichtling (TS) waves or crossflow (CF) instability. Following the initial linear growth processes, nonlinearity and subsequent secondary instabilities ${ }^{5}$ lead to the final breakdown of the flow to a turbulent state. Thus, receptivity modelling in stability analysis is a key requirement for making accurate transition predictions, since the initial disturbance magnitude is intrinsically linked to where, how and what type of nonlinear processes set in and dictate the type of flow transition.

Several theoretical studies concerning the receptivity of crossflow to surface roughness have been conducted ${ }^{6-9}$. Using compressible Linearised Navier-Stokes (LNS) equations, Collis and Lele ${ }^{10}$ investigated the receptivity on the leading-edge of a swept wing. Surface curvature, and more crucially non-parallel effects, were found to play a significant role in their receptivity calculations. Later, Wassermann and Kloker ${ }^{11}$ developed a vorticity formulation to explore the effects of nonlinearity in an incompressible flow over a flat plate, while the effects of freestream turbulence and wall roughness were examined by Schrader and co-workers ${ }^{12,13}$.

Crossflow disturbances on a swept wing, excited by localised roughness, were investigated experimentally by Saric at al. ${ }^{14-16}$, while numerical methods were utilised to replicate these particular experiments ${ }^{17,18}$. Isolated roughness elements centred about the location for neutral stability were found to provide the strongest receptivity response and hence influence in the transition process, while a more weaker response was observed for those elements located further downstream of the neutral location ${ }^{16}$. $\mathrm{Ng}$ and Crouch $^{17}$ implemented a finite Reynolds number residue approach, based on a local parallel flow assumption, and obtained qualitatively similar receptivity results as that achieved experimentally. Using a combination of direct numerical simulations and parabolised stability equations (PSE) methods, Tempelmann et al. ${ }^{18}$ found that the amplitude of the crossflow disturbance was about $40 \%$ of the experimental measurements and concluded that uncertainties in the experimental set-up were possibly responsible for the differences in the calculations.

A Monte-Carlo based uncertainty quantification (UQ) analysis was devised by Mughal and Ashworth ${ }^{19}$ using a harmonic LNS ${ }^{20}$ method. The flow used in their receptivity calculations was based on experimental measurements,

a)c.thomas@imperial.ac.uk 
while disturbances were excited by modelling strips of distributed surface roughness fields whose characteristics were derived from real measured painted and un-painted aluminium surfaces typically used on aircraft wings. Mean or expected receptivity amplitudes were computed for stationary crossflow disturbances, with both surface curvature and nonlinear effects included in their analysis. A maximum mean receptivity amplitude was attained for finitely long roughness strips located near the leading-edge of the model, while longer roughness distributions in the streamwise direction were found to be ineffective in further strengthening the fully developed crossflow disturbance. Furthermore, the variance of the mean receptivity predictions, could be used in nonlinear PSE simulations to quantify the variance in the onset of highly nonlinear interactions. The justification of using such an approach, is the recognition that surface roughness (however small) exists over the entire aerodynamic surface, and will comprise an element of machined in and a stochastic roughness variation. The most that a high-fidelity physics based receptivity-transition prediction approach can provide is the most likely transition location and an estimate of its' variance; the Monte-Carlo UQ based approach is ideal for this goal.

Our primary concern relates to outlining the most efficient means of undertaking the Monte-Carlo type analysis conducted by Mughal and Ashworth ${ }^{19}$, who used the direct LNS route. The adjoint linearised Navier-Stokes (ALNS) route is naturally suited for this purpose. Adjoint equations require that solutions be expanded as a bi-orthogonal set of eigensolutions ${ }^{21}$, and are advantageous over alternative receptivity schemes (i.e direct LNS) as they can be used to instantaneously predict the initial size of a disturbance to many environmental mechanisms. Hence, adjoint formulations are economical with both computational and time resources, and can be employed to conduct rapid and extensive Monte-Carlo type analysis. Hill ${ }^{22}$ first utilised the ALNS formula in the context of the two-dimensional (2D), parallel Blasius boundary layer, and carried out a thorough parametric study on the optimum conditions for generating TS wave disturbances. Non-parallel receptivity effects of the Blasius flow were examined using adjoint parabolised stability equations (APSE) ${ }^{23}$, while Dobrinsky and Collis ${ }^{24,25}$ extended the analysis to include the full spectrum of Falkner-Skan flows. The APSE for a quasi three-dimensional compressible flow were derived by Pralits at al. ${ }^{26}$, with results suggesting that three-dimensional (3D) disturbances are more sensitive than the corresponding 2D instabilities. Adjoint receptivity methods have been applied to a number of flow systems and boundary layer instabilities ${ }^{27-32}$, while Carpenter et al. ${ }^{33}$ examined crossflow receptivity in the context of a 3D boundary layer and a swept aerofoil. A review of adjoint methods and their many applications are given by Luchini and Bottaro ${ }^{34}$.

The remainder of this paper is structured as follows. In the subsequent section we describe the amplitude of a disturbance relating to the receptivity to external forces. The receptivity formulation based on the adjoint linearised Navier-Stokes equations is described in $\S I I I$. The ALNS is then utilised in $\S I V$ to explore the receptivity of crossflow in a compressible infinite swept wing boundary layer. In section $\S \mathrm{V}$, the Monte-Carlo type analysis is undertaken to determine the mean amplitude of crossflow disturbances generated by randomly distributed surface roughness. Conclusions are presented in $\S \mathrm{VI}$.

\section{AMPLITUDE OF A DISTURBANCE}

Consider a compressible non-dimensional flow $\mathbf{Q}_{\mathbf{B}}(x, y)=\left\{P_{B}, \mathbf{U}_{\mathbf{B}}, T_{B}\right\}(x, y)$ that develops in an infinite swept wing boundary layer. Here $\mathbf{U}_{\mathbf{B}}=\left\{U_{B}, V_{B}, W_{B}\right\}, P_{B}$ and $T_{B}$ respectively denote the undisturbed velocity, pressure and temperature fields in a surface-fitted coordinate system $\mathbf{x}=\{x, y, z\}$. The $y$ - and $z$-axes respectively denote the wall-normal and spanwise directions, whilst the $x$-axis represents the direction normal to the leading-edge of the wing where the position along the surface is measured from the attachment point $x=0$ (as depicted in figure 1). Perturbations $\overline{\mathbf{q}}=\{\bar{p}, \bar{u}, \bar{v}, \bar{w}, \bar{T}\}$ to $\mathbf{Q}_{B}$ are assumed to be infinitesimally small and linear:

$$
\overline{\mathbf{q}}(\mathbf{x}, t)=\mathbf{q}(x, y) \exp \{i(b z-\omega t)\}+\text { c.c. },
$$

where $\omega$ and $b$ respectively denote the non-dimensional periodic frequency and spanwise wavenumber of the disturbance. Additionally, we assume that the function q can be separated into two components that respectively describe the amplitude and amplification rate of the perturbation

$$
\mathbf{q}(x, y)=A \mathbf{q}^{\prime}(x, y) .
$$

The spatial-temporal evolution of a disturbance is then characterised by the function $\mathbf{q}^{\prime}$. For instance, the spatial development of a TS wave on a flat plate is generally represented as

$$
\mathbf{q}^{\prime} \sim \tilde{\mathbf{q}}(y) \exp \{i \alpha x\}
$$

where $\alpha$ is the complex wavenumber along the chord direction. The variation of $\mathbf{q}^{\prime}$ is governed by the flow conditions, including the freestream parameter settings and periodic perturbation assumptions. However, it does not take into 
(a)

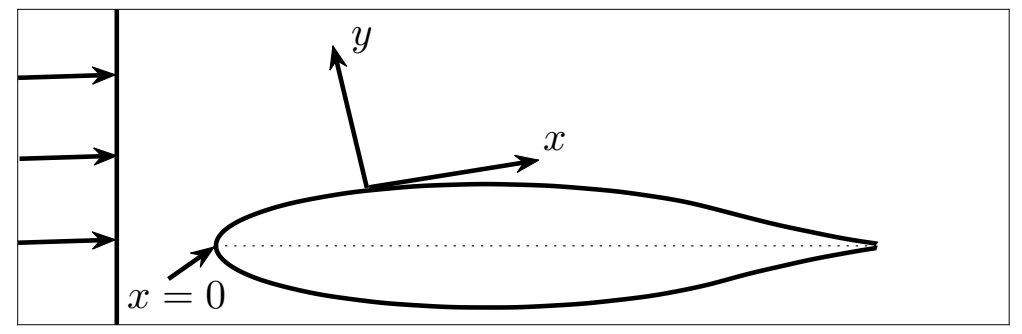

(b)

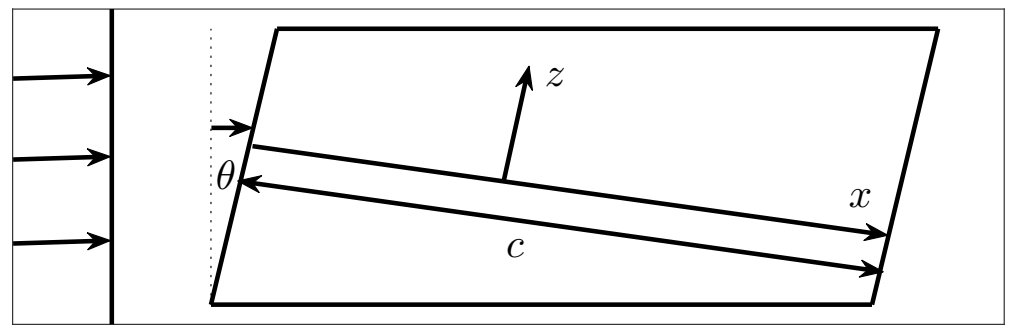

FIG. 1. Cross-sectional and aerial illustration of the infinite swept wing model.

account the scale or order of magnitude of the disturbance generated by the external forces. Surface roughness on a wing body can vary quite significantly, which may have considerable implications for the initial size of the perturbation and the onset of transition to turbulence. As a means of including these effects into the development of the boundary layer disturbances, we define $A$ in (1b) as being the receptivity amplitude, which describes the initial order of magnitude of the TS or crossflow instability. The size of $A$ is then influenced by the shape, form and level of the surface roughness or other external forcing. It is this quantity that forms the focal point of our subsequent analysis. How can we compute $A$, accurately and efficiently? How does it vary with curvature and compressible flow effects? What are the expected or mean orders of magnitude of crossflow disturbances excited by variable patches of roughness? These are just some of the questions that we intend to discuss and answer in the following sections.

\section{RECEPTIVITY FORMULATION}

The derivation of the adjoint method for predicting the receptivity of disturbances to roughness or other external forces has been covered in depth by Hill ${ }^{22}$ amongst several other authors. Thus, for purposes of brevity we only discuss the salient points of the methodology herein.

\section{A. Linearised Navier-Stokes (LNS) equations}

The ultimate goal of this study is to derive and utilise the adjoint equations, in order to undertake a fast receptivity analysis of crossflow disturbances to randomly distributed surface roughness. Although our primary interest concerns receptivity in compressible flows, in the subsequent discussion we only present the formulation for incompressible flow. This is to minimise description of the method and avoid introducing additional compressible flow parameters that may distract the reader from the fundamental details. The full compressible model (see appendices) can be formulated by including the energy equation for the temperature perturbation field into the following numerical derivation.

The incompressible LNS equations for perturbations $\overline{\mathbf{q}}=\{\bar{p}, \overline{\mathbf{u}}\}$ are given as

$$
\frac{\partial \overline{\mathbf{u}}}{\partial t}+\mathcal{L}\left(\mathbf{U}_{\mathbf{B}}\right) \overline{\mathbf{q}}=0
$$




$$
\nabla \cdot \overline{\mathbf{u}}=0
$$

where

$$
\mathcal{L}\left(\mathbf{U}_{\mathbf{B}}\right) \overline{\mathbf{q}}=\left(\mathbf{U}_{\mathbf{B}} \cdot \nabla\right) \overline{\mathbf{u}}+(\overline{\mathbf{u}} \cdot \nabla) \mathbf{U}_{\mathbf{B}}+\nabla \bar{p}-\frac{1}{R e} \nabla^{2} \overline{\mathbf{u}},
$$

for the locally defined Reynolds number $R e=U \delta / \nu$. The variables $U$ and $\delta$ denote the velocity and length scalings, while $\nu$ is the kinematic viscosity of the fluid.

\section{B. Adjoint Linearised Navier-Stokes (ALNS) Equations}

Introducing adjoint perturbation fields $\overline{\mathbf{q}}^{*}=\left\{\bar{p}^{*}, \overline{\mathbf{u}}^{*}\right\}$, the ALNS system of equations are derived from the Lagrange identity, which is formulated by applying integration by parts to the scalar product of (2) and $\overline{\mathbf{q}}^{*}$ :

$$
\left[\left(\frac{\partial \overline{\mathbf{u}}}{\partial t}+\mathcal{L}\left(\mathbf{U}_{\mathbf{B}}\right) \overline{\mathbf{q}}\right) \cdot \overline{\mathbf{u}}^{*}+\nabla \cdot \overline{\mathbf{u}} \bar{p}^{*}\right]+\left[\overline{\mathbf{u}} \cdot\left(\frac{\partial \overline{\mathbf{u}}^{*}}{\partial t}+\mathcal{L}^{*}\left(\mathbf{U}_{\mathbf{B}}\right) \overline{\mathbf{q}}^{*}\right)+\bar{p} \nabla \cdot \overline{\mathbf{u}}^{*}\right]=\frac{\partial K_{t}}{\partial t}+\nabla \cdot\left\{K_{x}, K_{y}, K_{z}\right\}
$$

where

$$
\mathcal{L}^{*}\left(\mathbf{U}_{\mathbf{B}}\right) \overline{\mathbf{q}}^{*}=\left(\mathbf{U}_{\mathbf{B}} \cdot \nabla\right) \overline{\mathbf{u}}^{*}-\left(\nabla \mathbf{U}_{\mathbf{B}}\right)^{\top} \overline{\mathbf{u}}^{*}+\nabla \bar{p}^{*}+\frac{1}{R e} \nabla^{2} \overline{\mathbf{u}}^{*}
$$

The $\mathbf{K}=\left\{K_{t}, K_{x}, K_{y}, K_{z}\right\}$ term on the right-hand-side of the Lagrange identity (4) represents the bi-linear concomitant, which is subsequently used to establish a formula for predicting the receptivity amplitude of a disturbance. (The total form of $\mathbf{K}$ for an incompressible flow with curvature effects is defined in the appendices). The ALNS equations are then given as

$$
\begin{gathered}
\frac{\partial \overline{\mathbf{u}}^{*}}{\partial t}+\mathcal{L}^{*}\left(\mathbf{U}_{\mathbf{B}}\right) \overline{\mathbf{q}}^{*}=0, \\
\nabla \cdot \overline{\mathbf{u}}^{*}=0
\end{gathered}
$$

\section{Normalisation}

In order to illustrate the effectiveness of the adjoint analysis in predicting the receptivity of convective disturbances, we assume that perturbations to the LNS system of equations (2) can be expanded as a single Fourier mode (1a), while the corresponding adjoint solution of $(6)$ is represented as

$$
\overline{\mathbf{q}}^{*}(\mathbf{x}, t)=\mathbf{q}^{*}(x, y) \exp \{-i(b z-\omega t)\}+\text { c.c. }
$$

A change in sign arises for $\omega$ and $b$ in the adjoint Fourier mode (7), since the adjoint perturbation evolves both backwards in time and spatially opposite sense to the LNS solution (1a).

If $\overline{\mathbf{q}}$ and $\overline{\mathbf{q}}^{*}$ are distinct modes of the respective systems (2) and (6), the Lagrange identity (4) can be reduced to the form

$$
\frac{\partial K_{x}}{\partial x}+\frac{\partial K_{y}}{\partial y}=0
$$

where $K_{x}$ and $K_{y}$ are given explicitly in terms of $\mathbf{q}$ and $\mathbf{q}^{*}$. Furthermore, (8) can be simplified by integrating over the $\{x, y\}$-parameter space, for $y \in[0: \infty)$ and $x \in\left[x_{1}: x_{2}\right]$, to give

$$
\mathcal{K}\left(\mathbf{q}, \mathbf{q}^{*}\right)=\int_{y=0}^{\infty} K_{x}\left(\mathbf{q}, \mathbf{q}^{*}\right) \mathrm{d} y=\text { constant }
$$

where the usual homogeneous boundary conditions are implemented on the surface and in the freestream. The quantity $\mathcal{K}$ is then conserved, which is a necessary requirement for the successful application of the adjoint treatment. Solutions $\mathbf{q}$ and $\mathbf{q}^{*}$ are then normalised such that

$$
\max _{z}|u(x, y)|=1 \quad \text { and } \quad \mathcal{K}=1 \quad \text { at } \quad x=x_{0},
$$

where $x_{0}$ is the chord location for neutral stability. 


\section{Formula for the Receptivity Amplitude}

Let us now consider the response of a disturbance to a source forcing at the wall that can either describe surface roughness or suction/blowing. The boundary conditions for the LNS system of equations (2) are given as

$$
\overline{\mathbf{u}}=\mathbf{u}_{w} \quad \text { on } \quad y=0 \quad \text { and } \quad \overline{\mathbf{u}} \rightarrow 0 \quad \text { as } \quad y \rightarrow \infty
$$

where $\mathbf{u}_{w}=\left\{u_{w}, v_{w}, w_{w}\right\}$ represents surface roughness

$$
u_{w}=-H U_{B, y}(x, 0), \quad v_{w}=\frac{\partial H}{\partial t}, \quad w_{w}=-H W_{B, y}(x, 0),
$$

or suction/blowing

$$
u_{w}=w_{w}=0, \quad v_{w}=H .
$$

Here $H=H(x) \exp \{i(b z-\omega t)\}$ describes the shape of the forcing.

Given the above boundary conditions, the Lagrange identity (4) may be recast after integrating with respect to $y \in[0: \infty)$, as

$$
\frac{\partial}{\partial x} \int_{y=0}^{\infty} K_{x}\left(\mathbf{q}, \mathbf{q}^{*}\right) \mathrm{d} y=\left.K_{y}\left(\mathbf{q}, \mathbf{q}^{*}\right)\right|_{y \rightarrow \infty} ^{y=0}
$$

where for an incompressible flow

$$
K_{y}=v_{w} p^{*}+\frac{1}{R e}\left(u_{w} \frac{\partial u^{*}}{\partial y}+w_{w} \frac{\partial w^{*}}{\partial y}\right)
$$

Assuming that the LNS perturbations are of the form (1a) coupled with (1b), equation (12) is integrated with respect to $x$ from $x_{1}$ through to $x_{2}$ that are located sufficiently far from any non-zero wall forcing. Hence, we obtain the formula

$$
A=\left.\int_{x_{1}}^{x_{2}} K_{y}\left(\mathbf{q}, \mathbf{q}^{*}\right)\right|_{y \rightarrow \infty} ^{y=0} \mathrm{~d} x
$$

Thus, the magnitude of a disturbance is established using only the form of the wall forcing and the normalised solutions of the ALNS system of equations. Thus, for a given set of flow conditions, we need only calculate the LNS and ALNS solutions once, which can then be used to determine the receptivity amplitude of a disturbance to any form of wall forcing. The above argument can be easily extended to include freestream mass or momentum sources, by following the terminology of $\mathrm{Hill}^{22}$.

\section{E. Numerical Method}

Both the LNS and ALNS systems of equations in harmonic form were discretised and solved in the manner described by Mughal ${ }^{19,20}$. Fourth-order accurate finite difference discretisation was implemented along the chordwise $x$-axis, while a pseudo-spectral approach was utilised in the wall normal $y$-direction. Solutions to the forced LNS and ALNS formulations were then obtained by utilising the lower-upper block factorisation matrix method.

\section{CROSSFLOW RECEPTIVITY ON AN INFINITE SWEPT WING}

\section{A. Base Flow}

We consider the steady compressible flow $\mathbf{Q}_{\mathbf{B}}$ that develops on an infinite swept wing, with an angle of sweep $\theta=25^{\circ}$ and a zero angle of inclination. Figure 1 illustrates the cross-sectional and aerial views of the model under consideration that was developed by Ashill et al. ${ }^{35}$. The swept wing geometry used in this investigation represents a simple aerofoil, but the receptivity analysis presented is general enough (in one form or other) to be applicable on a wide class of wing geometries, variable freestream conditions and surface roughness distributions. As the model is 
infinitely swept, the flow is independent of the spanwise $z$-direction; $\mathbf{Q}_{\mathbf{B}}$ depends only on the chord $x$ - and wall-normal $y$-directions. Thus, we need only consider the flow development in a two-dimensional plane that is normal to the wing leading-edge; i.e. along the $x$-axis.

The base flow $\mathbf{Q}_{\mathbf{B}}$ was obtained using two numerical schemes. Firstly, the flow was simulated using the TAU ${ }^{36}$ industrial flow solver. The method has previously been utilised by Thomas et al. ${ }^{37}$ to study the effects of waviness on the growth of crossflow disturbances. TAU establishes steady converged flow solutions by solving the formulation given by the Spalart-Allmaras-Edwards turbulence model ${ }^{38}$. However, for the subsequent study of laminar flow receptivity, the TAU formulation was reduced to the system of Navier-Stokes equations. This was achieved by setting the eddy viscosity (that appears in turbulent models) to zero. The Navier-Stokes equations were then solved subject to the no-slip conditions on the wing surface and the far-field boundary conditions given by pre-defined freestream specifications. Several inputs were initially specified, including but not limited to, the freestream Reynolds number $R e_{\infty}$ (measured per unit chord $c$ ) and the Mach number $M_{\infty}$. The TAU solver implements an explicit Runge-Kutta iterative scheme, where we assume a steady state solution is fully converged once residuals are of the order $10^{-6}$ and lower. To ensure the TAU formulation was utilised effectively, a sufficiently resolved mesh was constructed about the infinite swept wing. After careful experimentation it was determined that 40 mesh points across the boundary layer and approximately 300 mesh points along the wing surface were sufficient to capture accurate flow dynamics including the surface pressure variation. Beyond the boundary layer, the mesh density was significantly reduced while the far field limit was set at about 50 chord lengths from the wing. Increasing the number of mesh points beyond that specified above was found to cause negligible further variations in the base flow and subsequent receptivity calculations.

Converged solution of the TAU flow solver, in the form of a surface pressure field $C_{p}$ distribution, was then fed into a compressible boundary layer (CoBL) solver $^{39}$, to re-compute more accurate boundary-layer velocity profiles and associated derivative fields required in the LNS and ALNS formulation. The boundary layer solutions in a surface fitted coordinate system, was initiated at the attachment-point $x=0$, with subsequent solutions downstream computed by a streamwise marching in $x$ procedure. Numerical discretisation is based on using a fully implicit second-order accurate three-point backward differencing scheme along the $x$-direction, whilst a two point secondorder accurate (Keller-Box ${ }^{40}$ ) method is utilised in the wall normal $y$-direction. The method has previously been employed to successfully undertake full compressible instability studies on infinite swept wing bodies ${ }^{19,20,37,41}$. For the subsequent LNS and ALNS receptivity analysis, the base boundary-layer profiles were interpolated and mapped on to the $\{x, y\}$-grid, where up to $N_{x}=16000$ points were used along the $x$ discretised direction, while 51 optimally distributed nodes, with clustering within the boundary-layer were determined to be sufficient in $y$ to generate accurate receptivity calculations. Although larger (in $y$ ) grid resolutions were investigated, only marginal differences in the LNS and ALNS solutions arose, this was insufficient to affect the receptivity computations to the graphical accuracy presented in this paper. Although not published or included here, to confirm correctness of the codes, comparisons were made against solutions of the velocity-vorticity scheme developed by Davies \& Carpenter ${ }^{42}$, for the idealised swept Hiemenz flow and excellent agreement was obtained for both stationary and non-stationary disturbances.

\section{B. Incompressible Flow Analysis}

A base flow $\mathrm{Q}_{\mathrm{B}}$ was generated for the freestream conditions $R e_{\infty}=5 \times 10^{6}$ and $M_{\infty}=0.7$. Although these particular conditions establish a compressible flow, the associated effects on the boundary layer instabilities are neglected in the following discussion, and are instead examined in later sections. This allows us to compare and draw conclusions regarding compressibility effects on the receptivity process.

\section{LNS Method}

The full incompressible LNS and ALNS equations are presented in the appendices. Stationary disturbances $(\omega=0)$ were established for several dimensional spanwise wavenumbers $\beta$ that is measured per unit metre, for

$$
\beta=b / \delta,
$$

where $\delta$ is a local measure of the boundary layer thickness that is dependant on the reference position used to undertake the stability analysis. Thus, the value of $b$ varies with the chosen reference location that is arbitrarily selected (note that this does not affect the stability calculations, as other parameters are scaled in a consistent manner). Hence, for the purposes of presenting stability calculations, all results are illustrated in terms of the dimensional value of $\beta$ that remains fixed.

Figure 2(a) depicts the maximum absolute amplitude of the $u$-velocity perturbation as a function of the chord $x$-direction, for four spanwise wavenumbers $\beta$, where the effects of surface curvature have been neglected. (The 
(a)

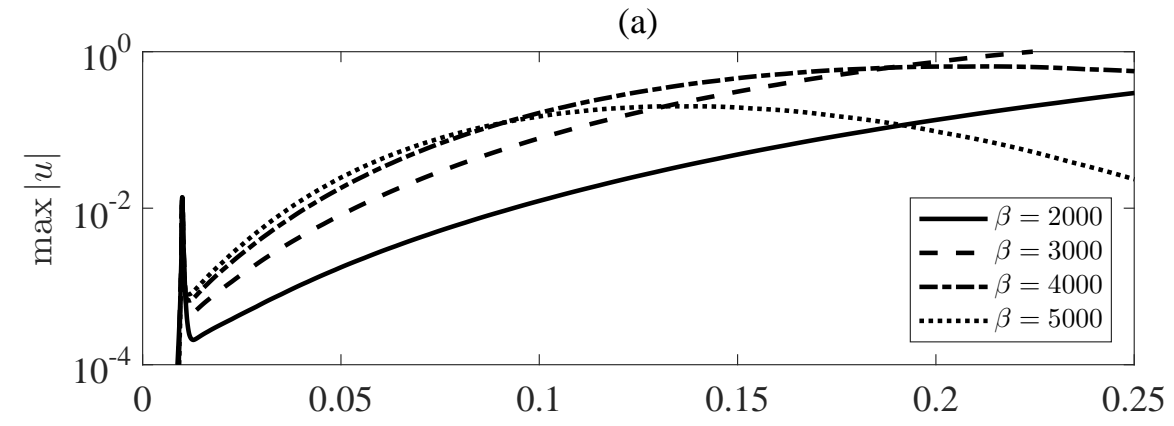

(b)

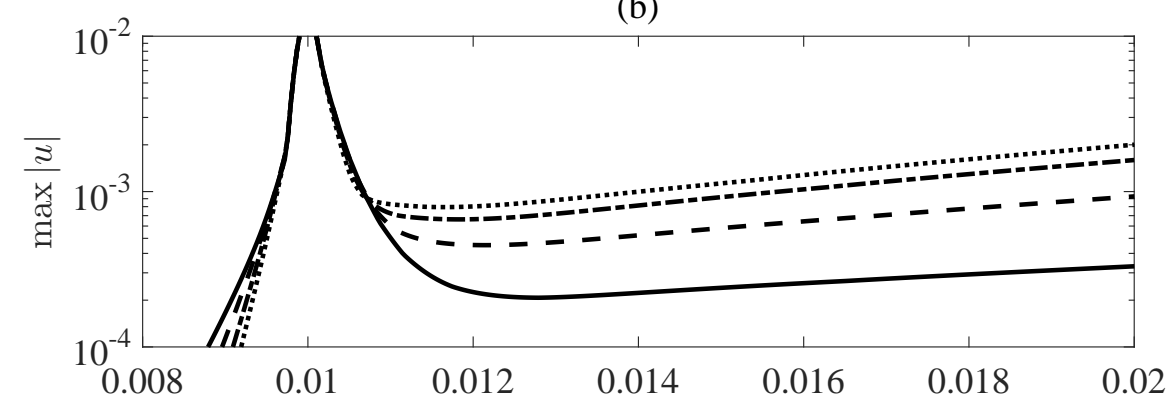

(c)

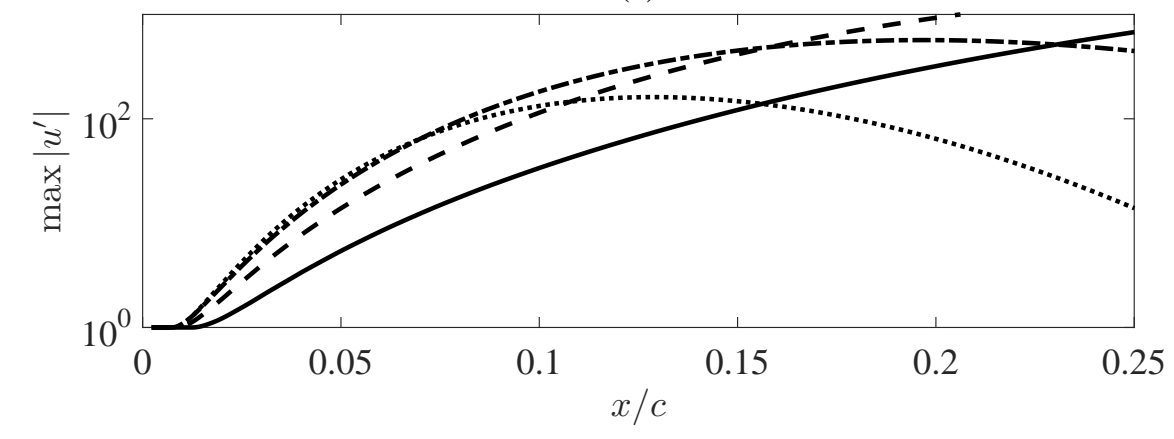

FIG. 2. (a, b) Maximum amplitude of the $u$-velocity perturbation excited by a stationary Gaussian roughness, centred about $x_{f}=0.01$ and variable spanwise periodicity $\beta$. Solutions are given for the incompressible LNS equations with curvature effects turned off and $\left\{R e_{\infty}, M_{\infty}\right\}=\left\{5 \times 10^{6}, 0.7\right\}$. (c) Exponential growth $u^{\prime}$ of the four perturbations.

curvature terms $\kappa$ and $\chi$ in the LNS and ALNS equations described within the appendices are respectively set to 0 and 1). The four disturbances were excited by a normalised Gaussian roughness distribution of the form

$$
H(x)=10^{-6} \exp \left\{-0.5\left(\left[x / c-x_{f}\right] / a\right)^{2}\right\} / \sqrt{2 \pi a^{2}},
$$

centred about $x_{f}=0.01$, for a constant variance $a=2 \delta$ and chord length $c$. Although the size of the Gaussian roughness remains constant for each $\beta$-simulation, there are significant variations in the response of the $u$-velocity perturbations. The obvious difference is related to the chordwise growth associated with each value of $\beta$. Near the leading-edge of the swept wing, stronger growth is associated with the larger $\beta$-calculations. However, as the disturbances develop downstream, the smaller $\beta$-perturbations achieve greater amplification rates.

A second observation, that is more pertinent to the current receptivity analysis, concerns the amplitude of the disturbance established by the Gaussian roughness. The region of interest is redrawn in figure 2(b) for a reduced chord range, while table I displays the corresponding receptivity amplitude $A$ for all four perturbations. The size of $A$ is estimated by assuming that crossflow perturbations are of the form (1) for

$$
u=A u^{\prime},
$$




\begin{tabular}{c|c|c|c}
$\beta\left(\mathrm{m}^{-1}\right)$ & In \& NCur & In \& Cur & Co \& Cur \\
\hline 2000 & $1.9 \times 10^{-4}$ & $1.7 \times 10^{-4}$ & $1.7 \times 10^{-4}$ \\
3000 & $3.2 \times 10^{-4}$ & $3.5 \times 10^{-4}$ & $3.5 \times 10^{-4}$ \\
4000 & $4.4 \times 10^{-4}$ & $5.2 \times 10^{-4}$ & $5.3 \times 10^{-4}$ \\
5000 & $5.6 \times 10^{-4}$ & $6.6 \times 10^{-4}$ & $6.7 \times 10^{-4}$
\end{tabular}

TABLE I. Receptivity amplitude $A$ associated with the disturbances excited by a Gaussian roughness centred about $x_{f}=0.01$. In - Incompressible, Co - Compressible, NCur - No Curvature and Cur - Curvature.

where the exponentially growing function $u^{\prime}$ is of the form utilised in PSE studies

$$
u^{\prime} \sim \exp \left\{\int_{x_{0}}^{x} \alpha\left(x^{\prime}\right) \mathrm{d} x^{\prime}\right\} .
$$

The magnitude of the $u^{\prime}$ component, corresponding to those disturbances depicted in figure 2(a, b) is drawn in 2(c). A constant receptivity amplitude was then obtained by scaling $u^{\prime}$, until it sits directly upon the results drawn in figure $2(\mathrm{a}, \mathrm{b})$. As the wall roughness is identical for all four disturbances, the size of $A$ must then be affected by the particular choice of $\beta$. For those cases illustrated, the largest amplitude $A$ is observed for $\beta=5000$, while $\beta=2000$ establishes the smallest amplitude. Clearly $A$ increases with increasing $\beta$, at least for the range of spanwise wavenumbers and the Gaussian roughness considered here. However, if we were then to consider the receptivity of disturbances to alternative forms of surface roughness or forcing, we would need to solve again the LNS system of equations and undertake a similar analysis to that described above. Of course, such a strategy would soon become very time consuming and monotonous, particularly if we were interested in conducting a statistical analysis on the receptivity of crossflow to many roughness distributions of variable dimensions. Furthermore, the spanwise wavenumber $\beta$, freestream conditions $\left\{R e_{\infty}, M_{\infty}\right\}$, surface curvature and compressibility can affect the receptivity calculations. Thus, to overcome the time constraints associated with the LNS scheme, we utilise solutions of the ALNS equations, to enable fast and accurate receptivity predictions.

\section{Adjoint Method}

Figure 3 depicts the absolute amplitude of the three normalised adjoint perturbation fields $\left|u_{y, y=0}^{*}\right| / R e,\left|w_{y, y=0}^{*}\right| / R e$ and $\left|p_{y=0}^{*}\right|$ that are required in the receptivity formula (14). These particular solutions correspond to the flow conditions $\left\{R e_{\infty}, M_{\infty}, \beta\right\}=\left\{5 \times 10^{6}, 0.7,4000\right\}$, where we have again ignored surface curvature and compressible flow effects. These three results can be used to understand and quantify several characteristics regarding receptivity to surface roughness and/or suction. Firstly, the three adjoint fields attain a maximum magnitude in the leading-edge region of the swept wing (about $x / c \approx 0.005$ ), which then decreases quickly, tending towards zero for larger $x$. Thus, we can predict that receptivity will be most amplified about the leading-edge region of the wing and that wall forcing further downstream will have a negligible impact on the size of the disturbance. Hence, if aircraft manufacturers want to minimise receptivity, the smoothness or level of surface roughness in the leading-edge region should be prioritised.

A second observation, regarding the results plotted in figure 3, relates to the relative sizes of the three adjoint perturbation fields and their role in the amplitude formula (14). For stationary only perturbations, receptivity to roughness is predicted by the sizes of the two terms $\left|u_{y, y=0}^{*}\right| / R e$ and $\left|w_{y, y=0}^{*}\right| / R e$ (which can be confirmed by substituting the no-slip condition (11b) into (14); solid and dashed lines), whilst the effect of suction forcing is estimated only by the magnitude of $\left|p_{y=0}^{*}\right|$ (substitute (11c) into (14); dotted line). Thus, for a roughness and suction distribution of identical size and form, we would expect the latter forcing mechanism to have a greater influence on the receptivity of the stationary crossflow instability.

Coupling the above adjoint solutions with the receptivity formula (14), we can then predict the amplitude $A$ of a disturbance to a Gaussian roughness distribution (15), instantaneously. Furthermore, as the method only requires a simple integration, we are able to compute $A$ for a range of forcing locations $x_{f}$ in a matter of seconds. Thus, the computational expense required for multiple LNS simulations is significantly reduced. Figure 4 displays the size of $A$ against the chord location $x_{f}$, for the four spanwise wavenumbers considered in figure 2. Receptivity to a Gaussian shaped roughness is strongest about $x_{f}=0.005$, as we may have expected given the results illustrated in figure 3 . Moreover, using the results drawn in figure 4, we are able to confirm the receptivity calculations tabulated in table I for the size of $A$ established by a Gaussian roughness centred about $x_{f}=0.01$.

Extending the above receptivity analysis of stationary disturbances to the wider spectrum of spanwise wavenumbers $\beta$, we were able to construct the $\{x / c, \beta\}$-contours illustrated in figure 5 . The freestream Reynolds and Mach 


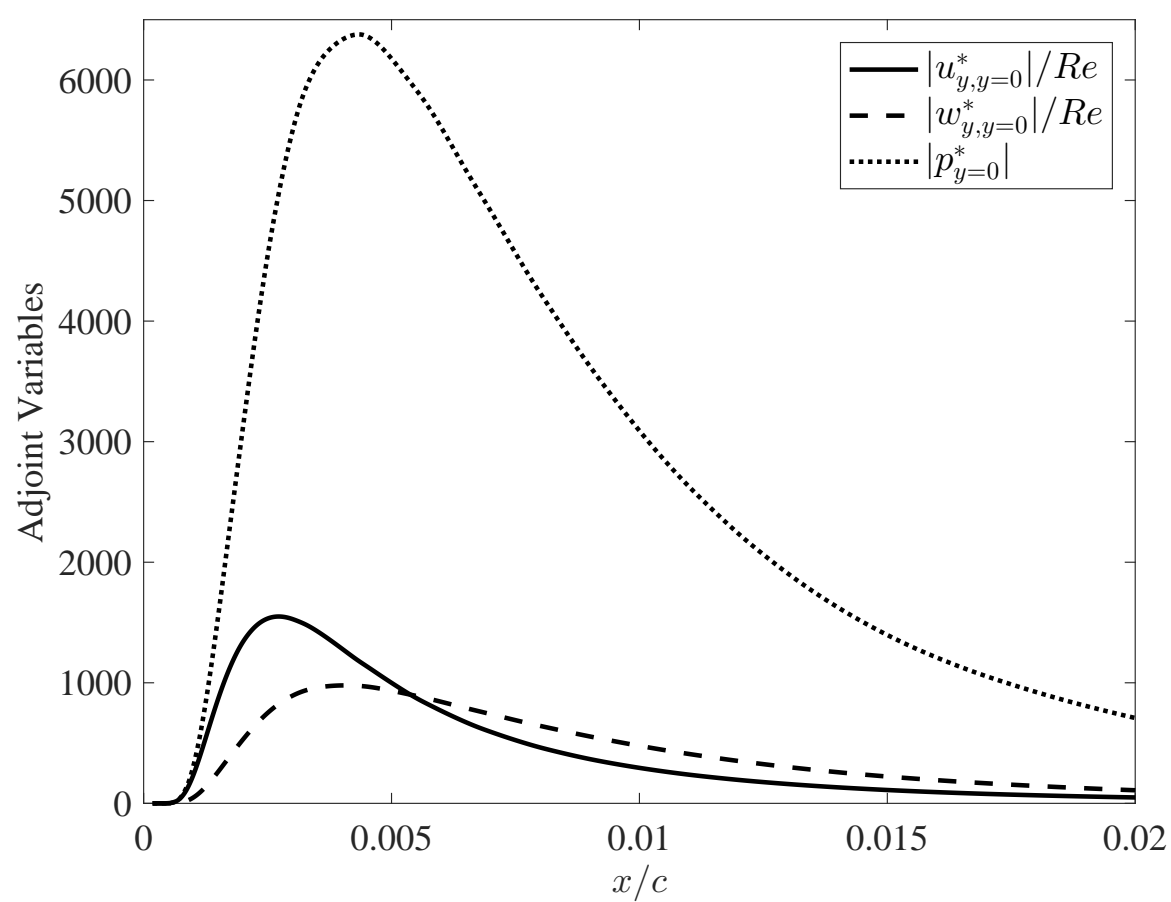

FIG. 3. Maximum amplitude of the three normalised adjoint perturbation fields $\left|u_{y, y=0}^{*}\right| / R e,\left|w_{y, y=0}^{*}\right| / R e$ and $\left|p_{y=0}^{*}\right|$ required for predicting receptivity, for $\left\{R e_{\infty}, M_{\infty}, \beta\right\}=\left\{5 \times 10^{6}, 0.7,4000\right\}$. Solutions are generated using the incompressible flow formulation with curvature effects turned off.

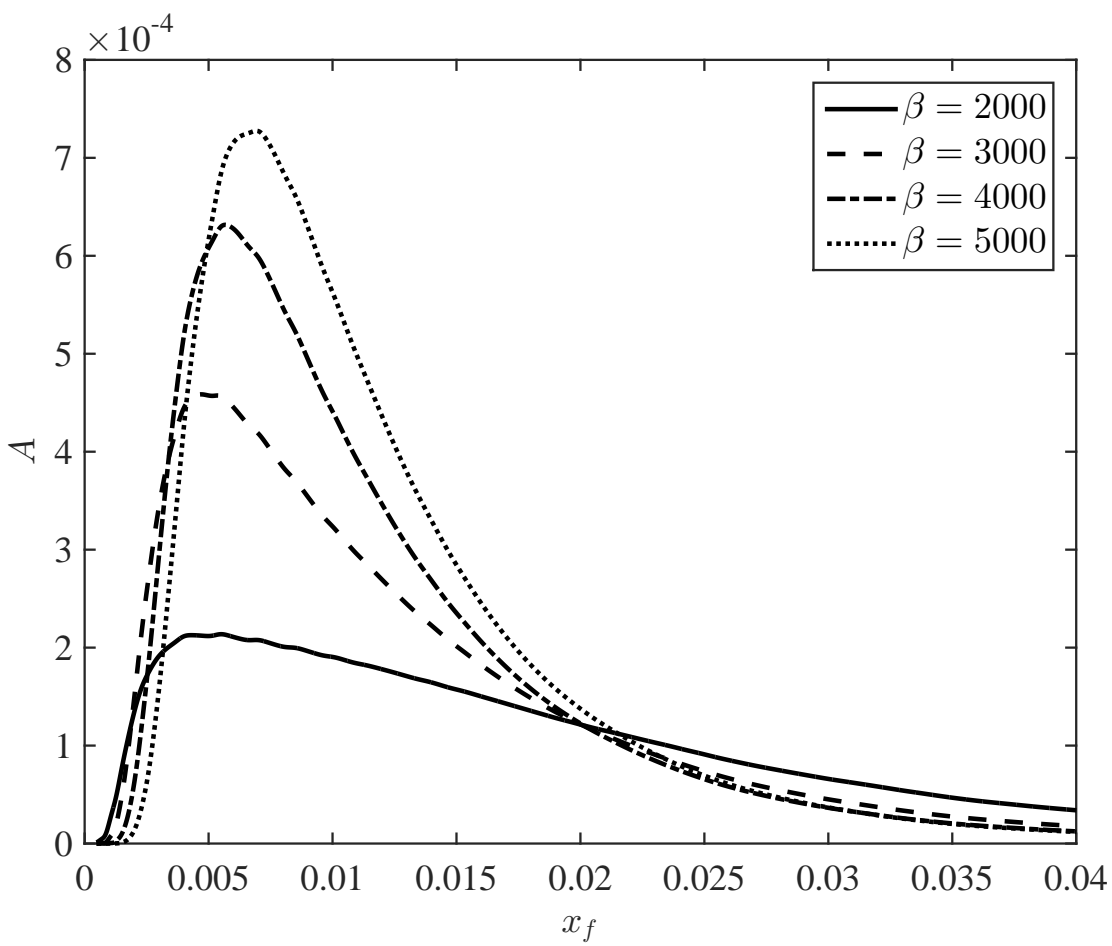

FIG. 4. Receptivity amplitude $A$ versus chord centre $x_{f}$ of a Gaussian roughness distribution (15). 
(a)

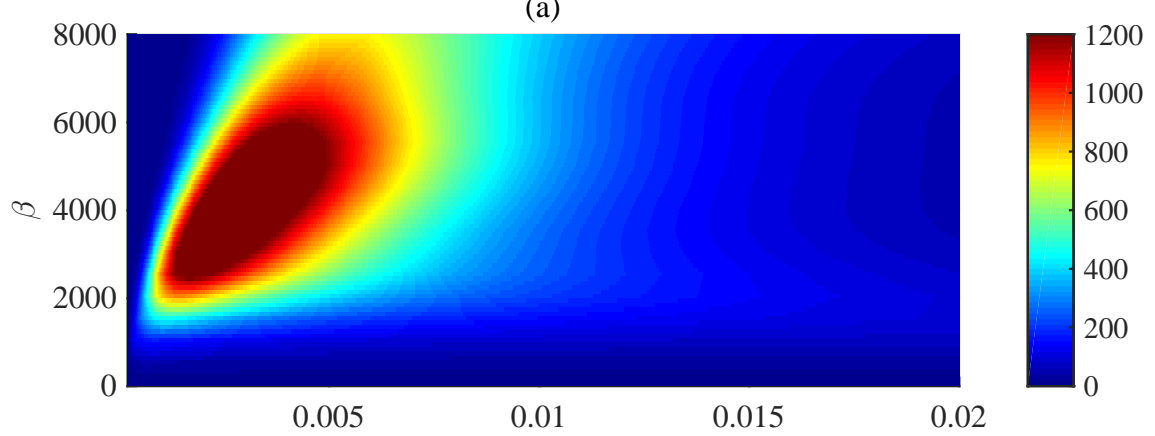

(b)

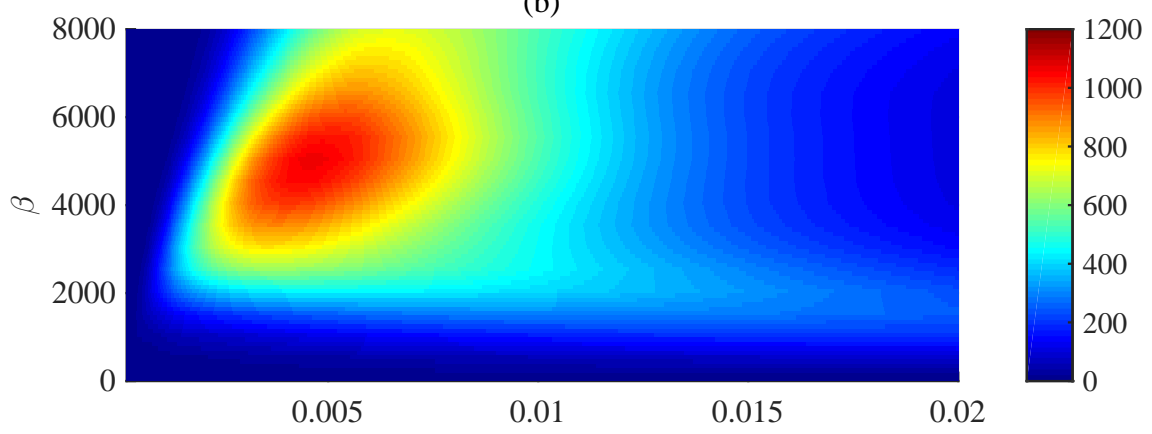

(c)

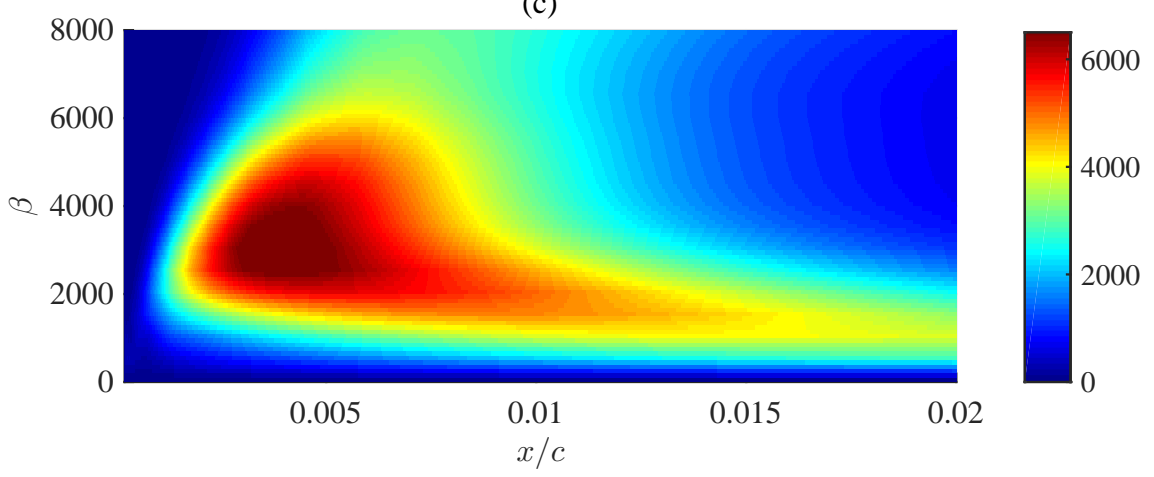

FIG. 5. Contours of the three normalised adjoint perturbation fields required for predicting receptivity, within the $\{x / c, \beta\}$ parameter space, for $\left\{R e_{\infty}, M_{\infty}\right\}=\left\{5 \times 10^{6}, 0.7\right\}$. Solutions are generated for the incompressible formulation with curvature effects turned off. (a) $\left|u_{y, y=0}^{*}\right| / R e ;(\mathrm{b})\left|w_{y, y=0}^{*}\right| / \operatorname{Re} ;$ (c) $\left|p_{y=0}^{*}\right|$.

number are unchanged from above. The three plots reiterate our earlier observations that the three adjoint fields are greatest near the leading-edge; receptivity is most affected by roughness or suction in this region. Additionally, we might anticipate that suction distributions generate disturbances with amplitudes about five times greater than that established by roughness. Furthermore, the contours suggest that receptivity is strongest for $\beta \in[4000: 5000]$, which we alluded to earlier in figure 2. Although this indicates that these particular modes may develop with a larger initial amplitude, they are not necessarily the instabilities responsible for transition, as this may yet be engineered by an alternative stronger growing disturbance. 
(a)

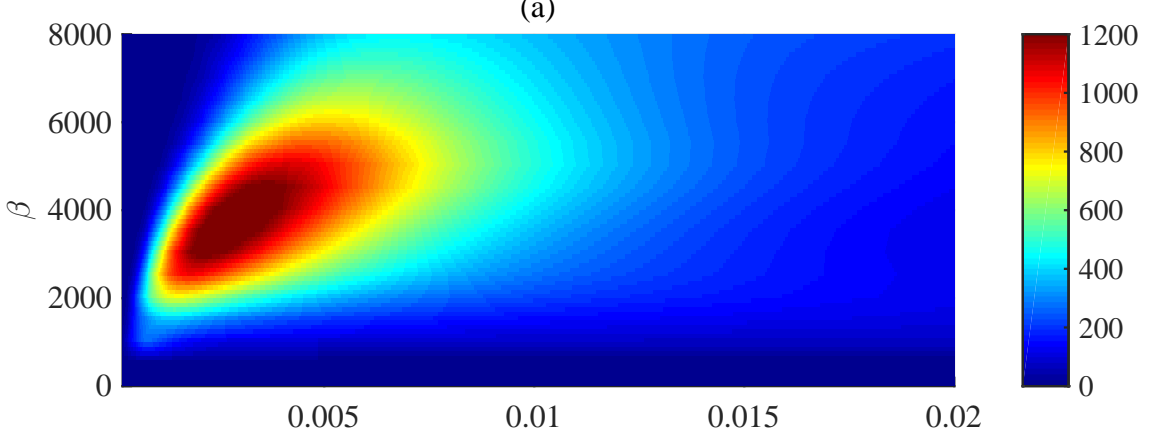

(b)

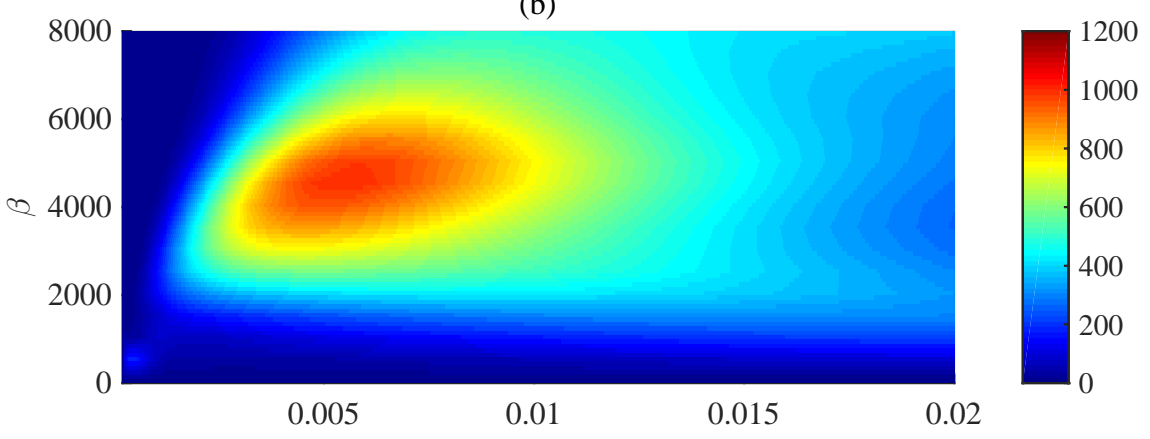

(c)

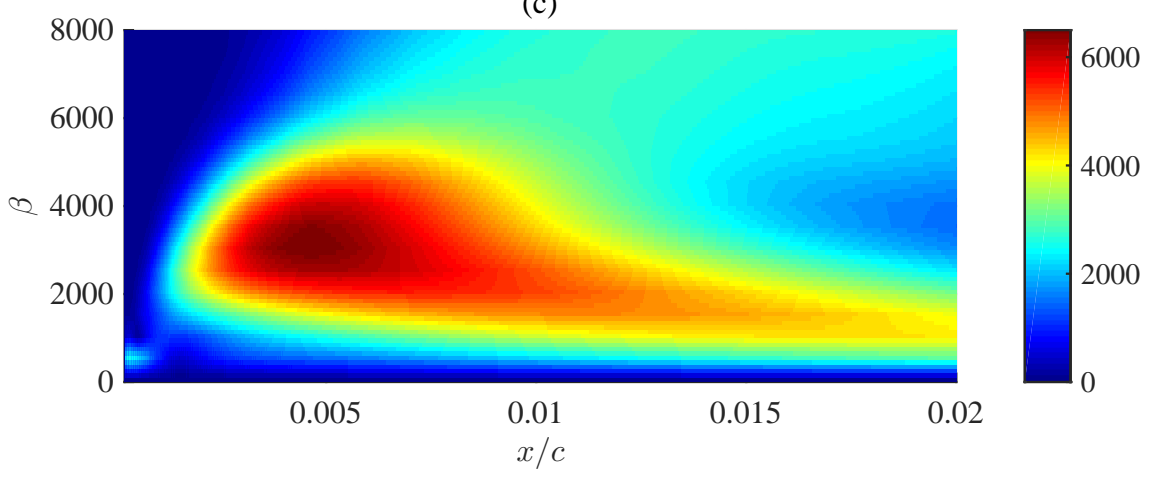

FIG. 6. Same as figure 5, but with curvature effects turned on.

\section{Curvature Effects}

The above analysis of incompressible flows is extended to include the effects of surface curvature. The body surface curvature terms $\kappa$ and $\chi$ that appear in the LNS and ALNS equations are assigned values given by the curvature of the infinite swept wing. Contours of the three adjoint perturbation fields required for predicting receptivity are constructed in figure 6 , by collating calculations of several $\beta$-simulations. The contour mapping, for each subplot, is the same as that given in figure 5, to allow easier comparison between the two sets of solutions. Qualitatively the pictures are similar to the contour illustrations drawn for the analysis without curvature effects; the adjoint perturbation fields are largest near the leading-edge. However, there would appear to be some small differences in the amplitude of each perturbation. The contour illustrations with curvature effects indicate that the maximum magnitude is smaller than those solutions generated without curvature; the intensity of the larger amplitude, dark red contours in figure 5, are greater than that depicted in figure 6. An additional difference, between the two sets of calculations, is that the curvature solutions appear to display magnitudes of an appreciable size further downstream. 
(a)

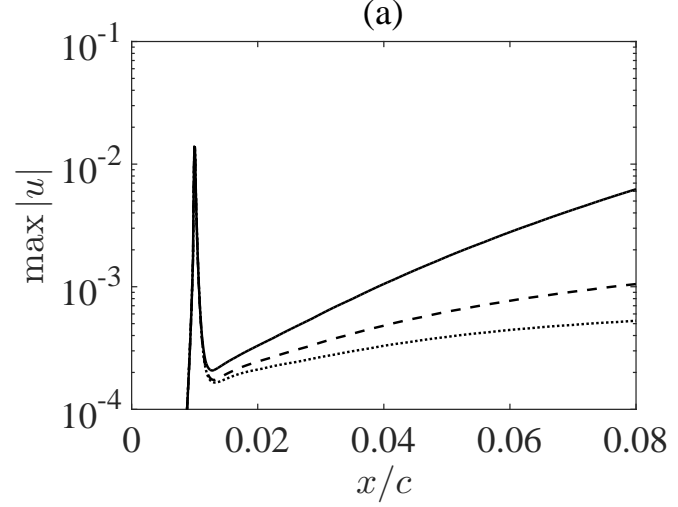

(c)

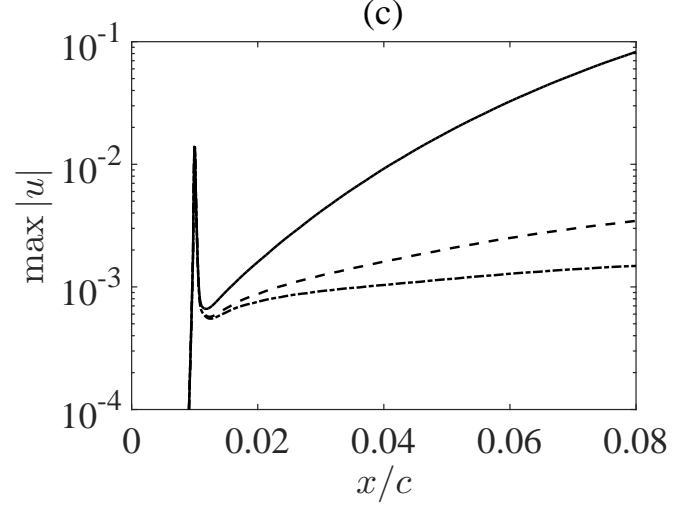

(b)

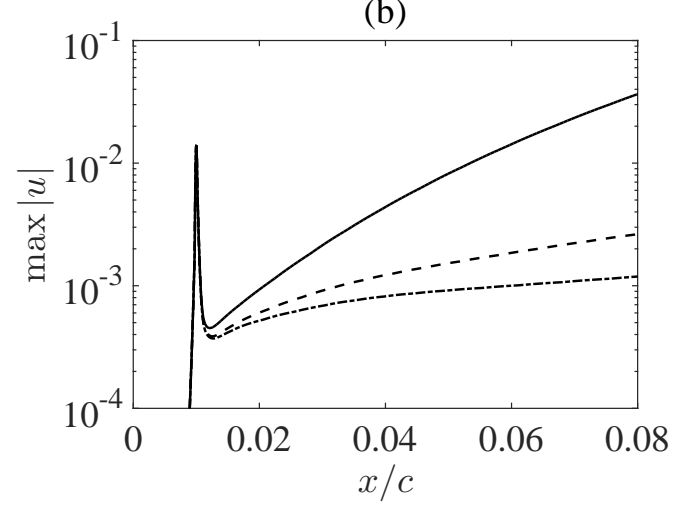

(d)

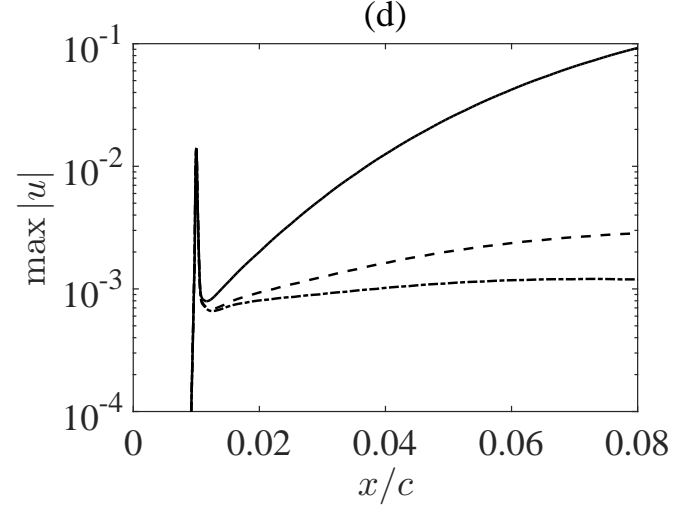

FIG. 7. Maximum amplitude of the $u$-velocity perturbation excited by a stationary Gaussian roughness, centred about $x_{f}=0.01$ and variable spanwise wavenumber $\beta$. Solid lines depict solutions of the incompressible scheme with curvature effects turned off, dashed lines illustrate incompressible calculations with curvature effects and dotted lines correspond to the fully compressible (curvature included) scheme. (a) $\beta=2000$; (b) $\beta=3000$; (c) $\beta=4000$; (d) $\beta=5000$.

This particular observation is best illustrated by the contour levels of amplitude 600 (green) in figure 6(a, b) that extend to larger chord positions than that drawn in figure $5(\mathrm{a}, \mathrm{b})$. Thus, receptivity may be affected by a greater chord section of the infinitely swept wing. However, the region of influence would still appear to be confined to the leading-edge.

The max $|u|$-velocity results depicted in figure 2(a) are redrawn in figure 7 (solid lines) and compared against the equivalent solutions generated with curvature effects (dashed). The dotted lines illustrate the corresponding computations established using the fully compressible LNS equations, which we will discuss further in the subsequent section. A Gaussian shaped roughness distribution is again used to excite the crossflow instabilities. Curvature is found to have a two-fold effect on the response of each disturbance. Firstly, the amplification rate of the crossflow instability is significantly damped by the introduction of curvature effects, whilst the receptivity amplitude $A$ is also affected by curvature; the initial size of the disturbance is reduced for the cases illustrated in figure 7 . However, assuming that the spatial development of crossflow with curvature can also be decomposed as (1b) for an exponentially growing function (16) (that is different to that established for perturbations without curvature effects), we find that the amplitude $A$ of the disturbance can increase with curvature, at least for a Gaussian roughness located about $x_{f}=0.01$ and $\beta \geq 3000$ (see table I).

Figure 8 reproduces the calculations depicted in figure 4, but with the inclusion of the surface curvature effects. The results indicate that over the chord range $0 \leq x_{f} \leq 0.01$, larger amplitudes $A$ are generally obtained for disturbances without curvature, while for $x_{f}>0.01$ solutions with curvature typically establish larger magnitudes. Thus, surface curvature is found to play both a significant role in establishing the true growth of the crossflow instability, and affects the receptivity amplitude of the disturbance. 

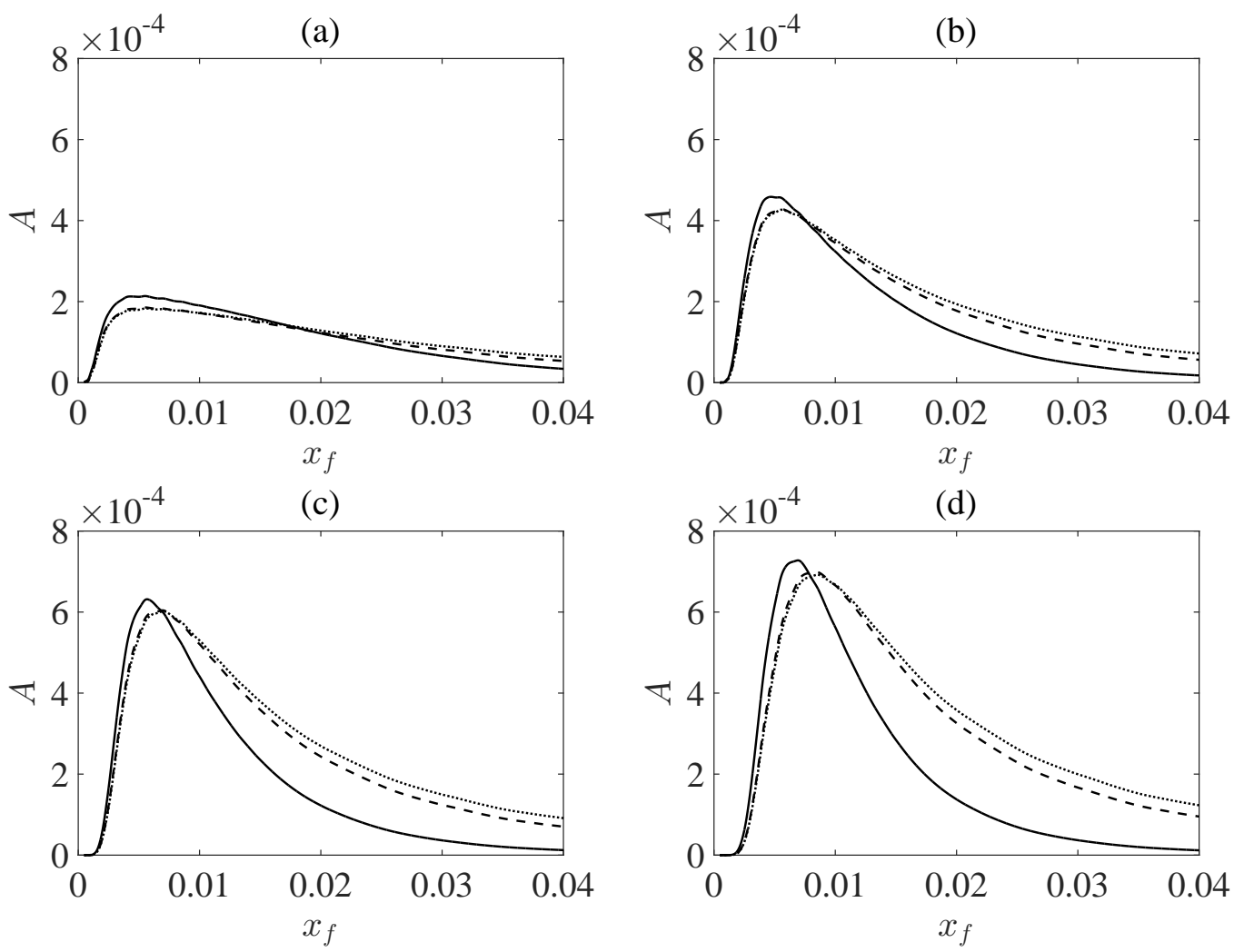

FIG. 8. Receptivity amplitude $A$ versus chord centre $x_{f}$ of a Gaussian roughness (15). Line types and flow conditions are the same as that given in figure 7 .

\section{Compressible Flow Analysis}

The inclusion of compressible flow effects in both the LNS and ALNS formulations are detailed within the appendices. Compressible perturbation solutions, matching those above for an incompressible flow, are drawn using dotted lines in figures 7 and 8. Growth rates of the crossflow instabilities are further reduced by the introduction of compressible flow effects. However, the size of the receptivity (or initial disturbance) amplitude to the Gaussian roughness distribution is not significantly different to that established for an incompressible flow with curvature effects. This is emphasised in table I, which documents the size of $A$ for all three flow schemes considered in figures 7 and 8 .

Figure 9 compares the magnitude of the three adjoint perturbation fields $\left|u_{y, y=0}^{*}\right| / R e,\left|w_{y, y=0}^{*}\right| / R e$ and $\left|p_{y=0}^{*}\right|$, computed using the three flow schemes with conditions $\left\{\operatorname{Re}_{\infty}, M_{\infty}, \beta\right\}=\left\{5 \times 10^{6}, 0.7,4000\right\}$. Solid and dashed lines respectively illustrate solutions generated using the incompressible flow without and with curvature effects, while dotted lines depict the corresponding fully compressible flow results. The results indicate that compressibility has a negligible effect on receptivity, even though the freestream Mach number of the flow under consideration reflects typical commercial flight conditions. The insensitivity to compressible effects is due to fact that the receptivity analysis is close to the leading edge region, where despite the rapidly accelerating flow velocity, local Mach numbers are still relatively low.

Table II tabulates the percentage absolute difference between the maximum of the adjoint perturbation fields, $\left|u_{y, y=0}^{*}\right| / R e$ and $\left|w_{y, y=0}^{*}\right| / R e$, established by the incompressible and compressible flow schemes with curvature effects. Results are presented for several Mach numbers, with variations ranging from 3\% to $5 \%$ for the largest valued $M_{\infty}$, while for $M_{\infty}=0.1$ the disparity is about $1 \%$. Thus, the results appear to suggest that compressible flow characteristics in LNS calculations (at least for the wing model and Mach numbers considered herein) are necessary for accurately computing the amplification rate of crossflow, but they do not seem to have an appreciable effect on the initial amplitude of the disturbance. 
(a)

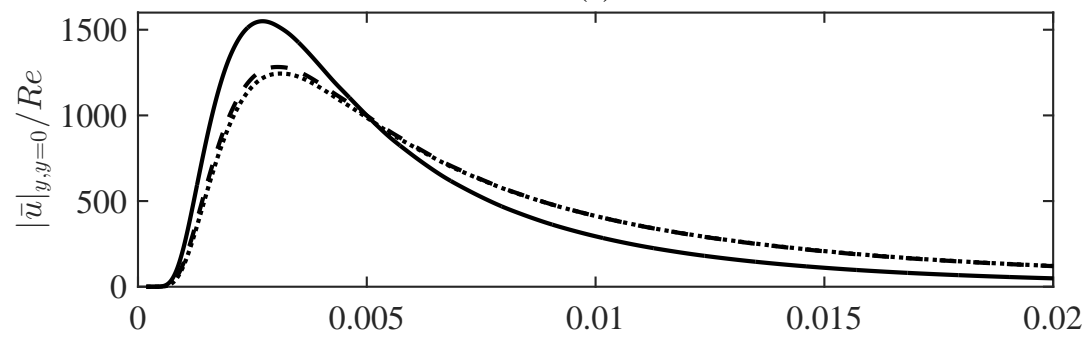

(b)

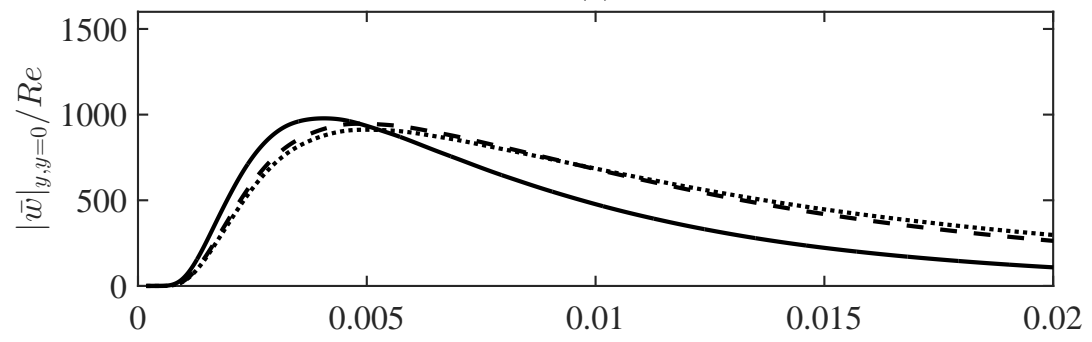

(c)

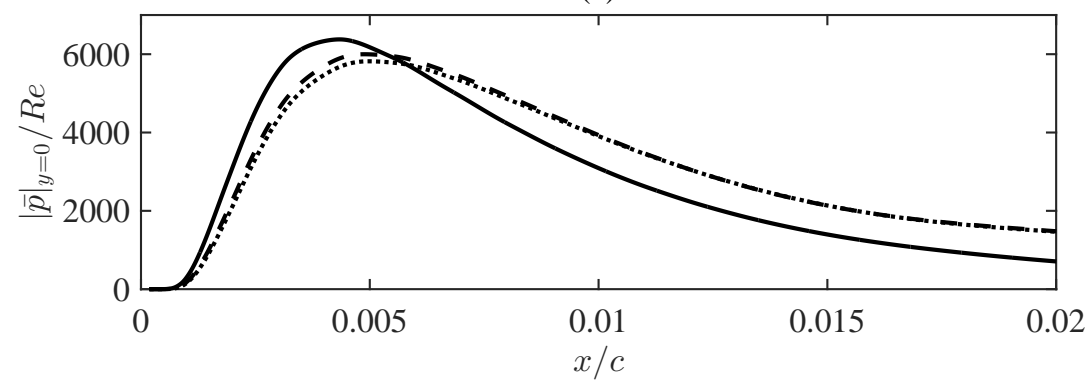

FIG. 9. Magnitudes of the three adjoint perturbation fields required for predicting receptivity for the flow conditions $\left\{R e_{\infty}, M_{\infty}, \beta\right\}=\left\{5 \times 10^{6}, 0.7,4000\right\}$. Solid lines depict solutions of the incompressible flow scheme with curvature effects turned off, dashed lines illustrate incompressible calculations with curvature effects and dotted lines correspond to the fully compressible flow with curvature formulation. (a) $\left|u_{y, y=0}^{*}\right| / R e ;(\mathrm{b})\left|w_{y, y=0}^{*}\right| / R e ;(\mathrm{c})\left|p_{y=0}^{*}\right|$.

\begin{tabular}{c|c|c|c|c} 
& \multicolumn{2}{|c|}{$\beta=4000 \mathrm{~m}^{-1}$} & \multicolumn{2}{|c}{$\beta=5000 \mathrm{~m}^{-1}$} \\
\hline$M_{\infty}$ & $\left|u_{y, y=0}^{*}\right| / R e$ & $\left|w_{y, y=0}^{*}\right| / R e$ & $\left|u_{y, y=0}^{*}\right| / R e$ & $\left|w_{y, y=0}^{*}\right| / R e$ \\
\hline 0.1 & 1.02 & 0.58 & 1.23 & 0.62 \\
0.3 & 2.12 & 1.07 & 2.01 & 1.85 \\
0.5 & 2.92 & 2.27 & 3.04 & 2.64 \\
0.7 & 4.79 & 3.84 & 5.42 & 4.37
\end{tabular}

TABLE II. Percentage difference (\%) in the maximum amplitude of $\left|u_{y, y=0}^{*}\right| / R e$ and $\left|w_{y, y=0}^{*}\right| / R e$, between solutions of the incompressible and compressible schemes with curvature effects turned on. The Reynolds number $\operatorname{Re}_{\infty}=5 \times 10^{6}$.

\section{E. Variable Freestream Reynolds and Mach Numbers}

The adjoint fields $\left|u_{y, y=0}^{*}\right| / R e$ and $\left|w_{y, y=0}^{*}\right| / R e$, required for predicting receptivity to surface roughness, are computed for a broad range of freestream Reynolds and Mach numbers. Solutions are established for variable $R e_{\infty}, M_{\infty}$ and $\beta$, and three-dimensional contour slices are constructed in figures 10 and 11 . In the former figure, variations in $M_{\infty}$ are depicted for $R e_{\infty}=5 \times 10^{6}$, while in the latter illustration $R e_{\infty}$ varies and $M_{\infty}=0.7$. The two sets of results reveal that increasing both the Mach and Reynolds number, increases the maximum absolute size of the two adjoint 


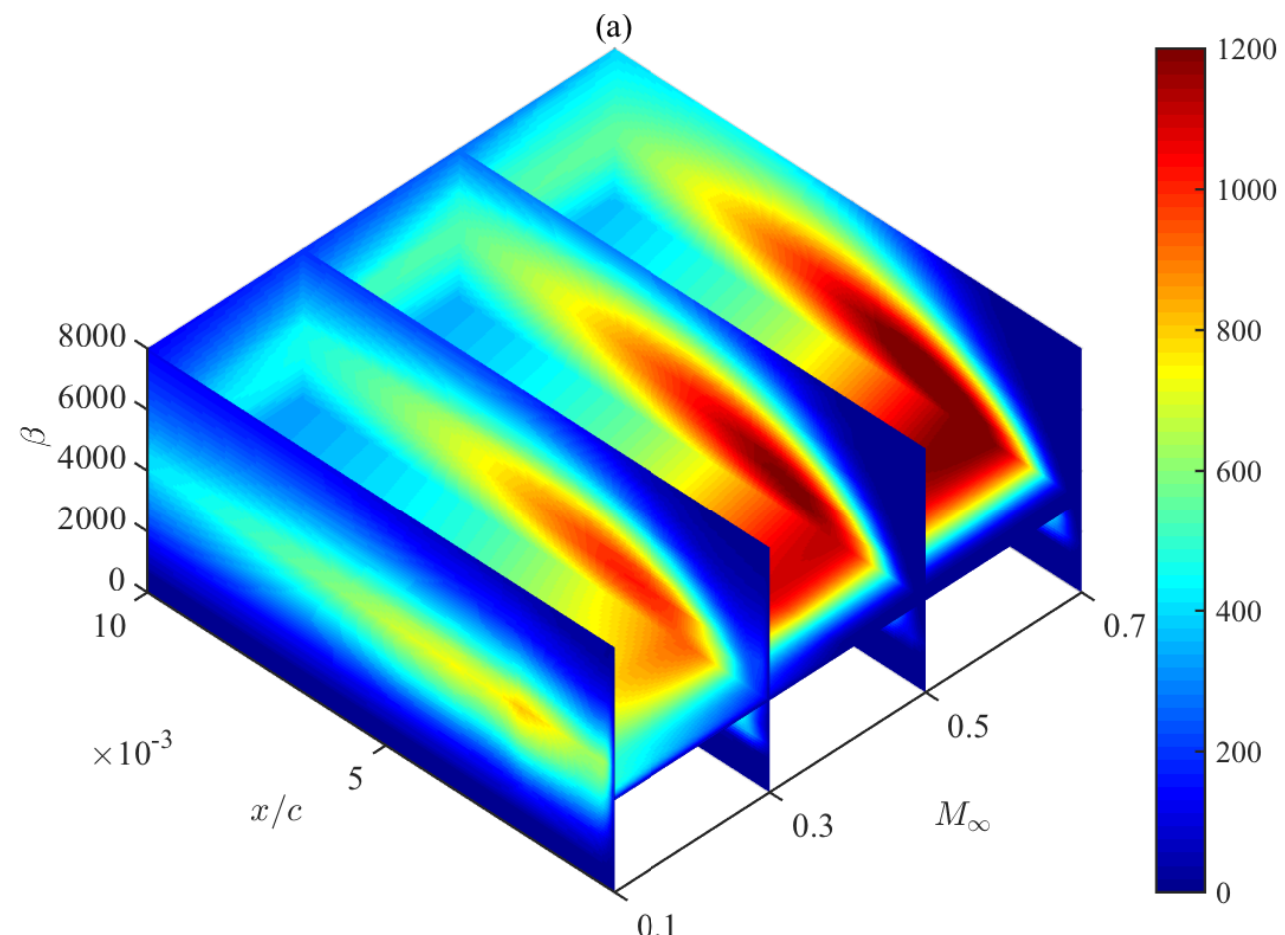

(b)

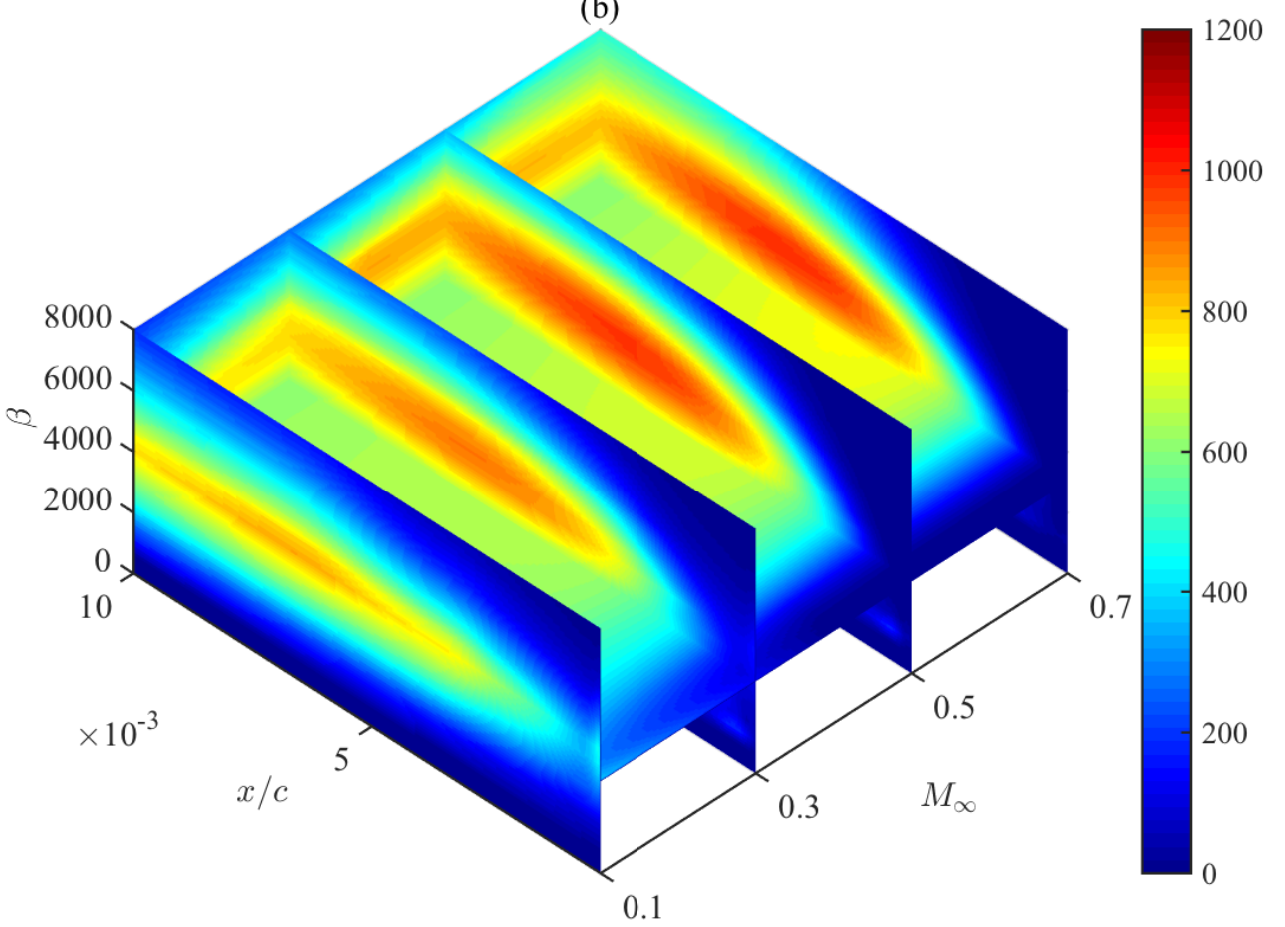

FIG. 10. Contour slices of (a) $\left|u_{y, y=0}^{*}\right| / R e$; (b) $\left|w_{y, y=0}^{*}\right| / R e$, for variable $\beta$ and $M_{\infty}$. Calculations are based on the fully compressible scheme with curvature effects and $R e_{\infty}=5 \times 10^{6}$. 

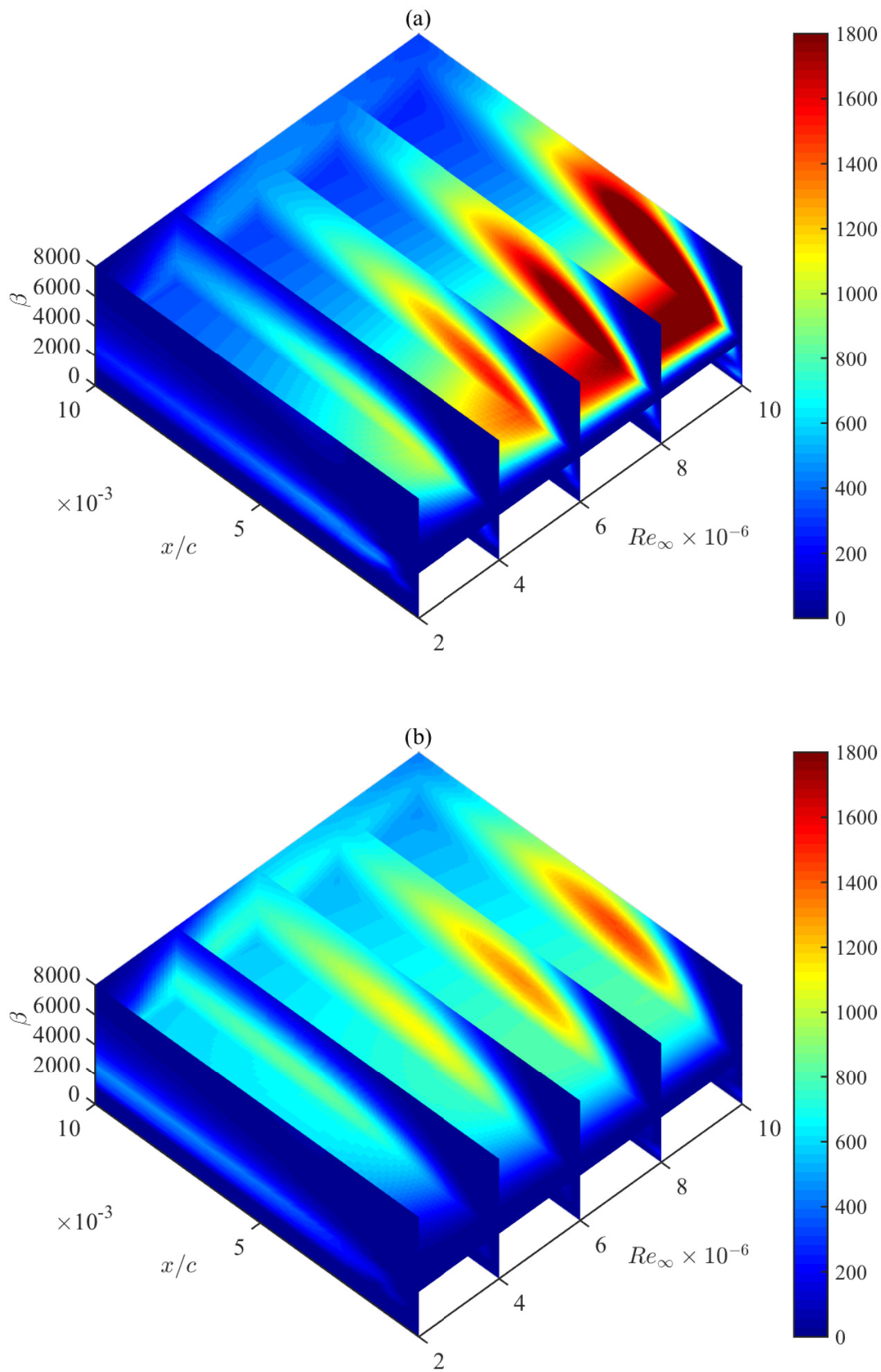

FIG. 11. Same as figure 10, but illustrating the variations in $R e_{\infty}$ and $M_{\infty}=0.7$. 
perturbation fields. This would then suggest that the receptivity amplitude $A$ of a disturbance excited by surface roughness will typically increase with both $M_{\infty}$ and $R e_{\infty}$. However, as shown in the subsequent analysis of randomly generated roughness, this is not necessarily the case, and smaller valued $R e_{\infty}$ flows can establish larger receptivity amplitudes.

\section{RANDOMLY DISTRIBUTED SURFACE ROUGHNESS}

\section{A. Model}

Thus far, we have considered the receptivity of stationary disturbances to simple 2D Gaussian roughness distributions. However, natural surface roughness is random and 3D. Furthermore, the surface will be subject to several distributed peaks and troughs of varying heights and wavelengths. In order to investigate the receptivity of crossflow to random 3D roughness, Mughal and Ashworth ${ }^{19}$ (using LNS method) implemented a simple model composed of many cosine series

$$
H_{3 D}(x, z)=\sum_{k=N_{1}}^{N_{2}} \sum_{j=N_{1}}^{N_{2}} a_{j k} \cos \left(2 \pi\left[j x / \lambda_{x}+k z / \lambda_{z}+\phi_{j k}\right]\right),
$$

where $\phi_{j k}$ represent randomly generated phase shifts and $a_{j k}$ roughness amplitudes derived from measured roughness surfaces. The parameters $\lambda_{x}$ and $\lambda_{z}$ denote wavelengths along the chord $x$ - and spanwise $z$-directions of the patch of roughness, while $N_{1}$ and $N_{2}$ respectively specify long and short scale wavelength filters.

For the subsequent receptivity analysis we assume that periodicity along the spanwise direction is retained and implement a simplification to the 3D model considered by Mughal and Ashworth. Although 3D features will be lost, a comprehensive analysis of receptivity to $2 \mathrm{D}$ random roughness can still reveal several meaningful characteristics. Random roughness is then modelled as

$$
H(x)=\sum_{j=N_{1}}^{N_{2}} a_{j} \cos \left(2 \pi\left[j x / \lambda_{x}+\phi_{j}\right]\right) .
$$

Figure 12 depicts a possible randomly generated roughness distribution of length $x_{l e n} \in\left[x_{s}: x_{e}\right]$, where in the subsequent analysis we set $x_{s}=0$ to ensure that the roughness starts upstream of the onset of the crossflow instability.

Characterising the level of the surface roughness is then achieved by defining a surface root-mean-square (rms)

$$
H_{\mathrm{rms}}=\sqrt{\frac{1}{x_{l e n}} \int_{0}^{x_{l e n}} H^{2} \mathrm{~d} x}
$$

which we use to scale the randomly generated roughness (18), so that $H_{\mathrm{rms}}$ is $0.1 \%$ of the local boundary layer thickness $\delta$.

\section{B. Linear Receptivity Analysis}

\section{Calculating Mean Amplitudes}

The random roughness model (18) is dependent on several parameters, which will influence the receptivity of crossflow. Additionally, for roughness strips with a fixed length, wavelength and filter settings, the magnitude of $A$ established by two randomly generated roughness distributions will not necessarily be the same. In fact they are likely to be very different. However, given the observations of previous investigators ${ }^{19}$, we might expect that a Monte-Carlo type analysis (where we compute the receptivity to many roughness strips) will establish a constant mean amplitude

$$
\mu=\frac{\sum_{s} A_{s}}{S}
$$

and a variance $\sigma$ after $S$ roughness simulations. As the adjoint based receptivity method only requires that the LNS and ALNS equations be solved once, we can undertake a Monte-Carlo analysis of many thousands of random roughness strips very quickly. Hence, the method overcomes the lengthy time simulations associated with undertaking a LNS receptivity investigation. 


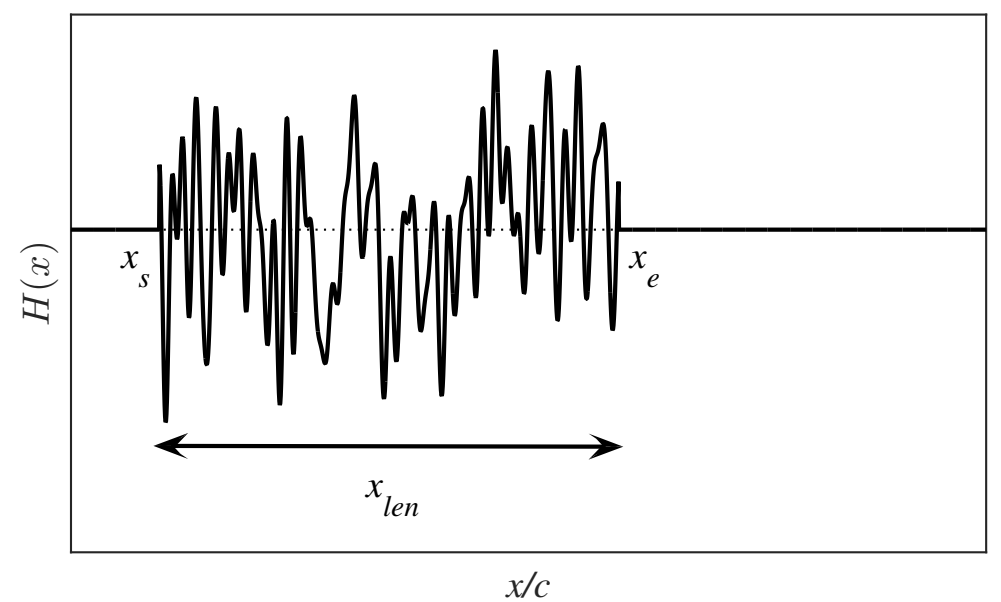

FIG. 12. Example of a randomly distributed roughness panel of length $x_{l e n}$.
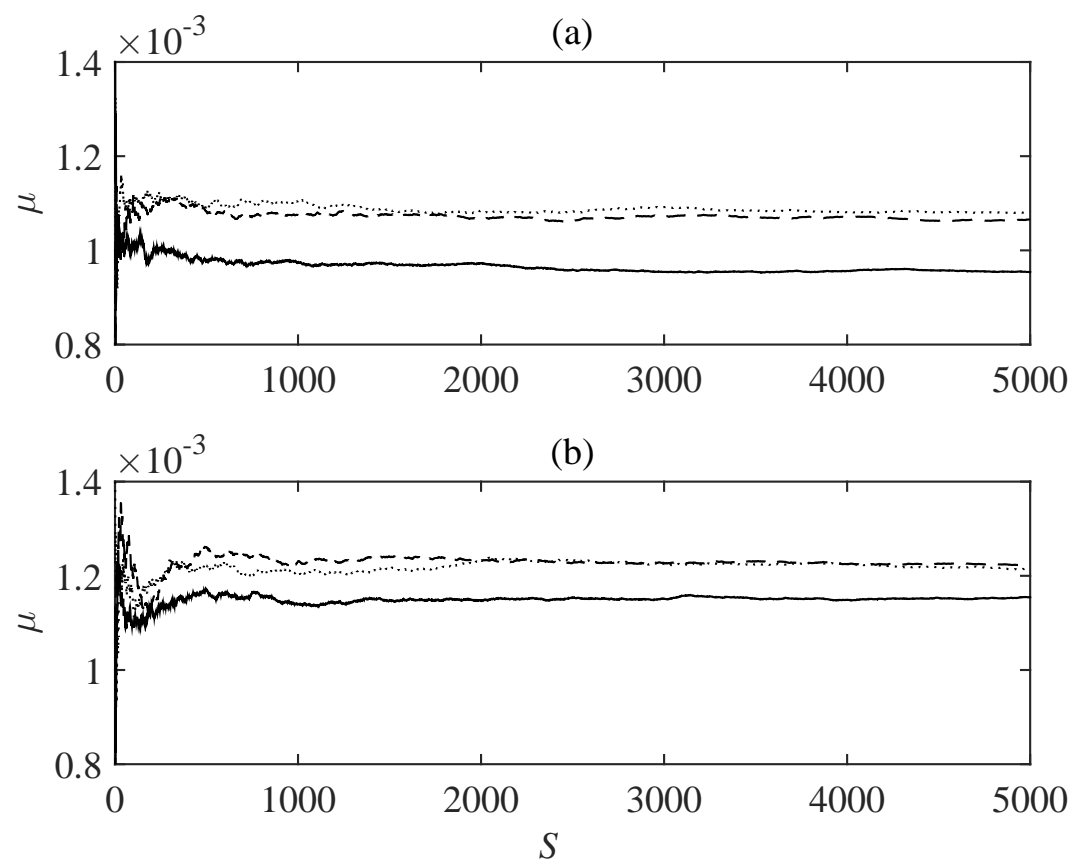

FIG. 13. Mean receptivity amplitude $\mu$ versus $S$ roughness field realisations, for $\left\{R e_{\infty}, M_{\infty}, \beta\right\}=\left\{5 \times 10^{6}, 0.7,4000\right\}$. Solid lines depict results for roughness panels $x_{l e n}=0.02$, dashed $x_{l e n}=0.04$ and dotted $x_{l e n}=0.06$. The wavelength $\lambda_{x}=0.2$ and the short wavelength filter (a) $N_{2}=80$, (b) $N_{2}=160$.

Figure 13 depicts the mean receptivity amplitude $\mu$ against the number of roughness simulations $S$, for roughness strips of variable length $x_{l e n}$ and short wavelength filter $N_{2}$. The flow conditions $\left\{R e_{\infty}, M_{\infty}, \beta\right\}=\left\{5 \times 10^{6}, 0.7,4000\right\}$, $\lambda_{x}=0.2$ and the long wavelength filter $N_{1}=1$ are fixed for much of the subsequent investigation. In all cases considered, the size of $\mu$ converges towards a constant (though not necessarily the same) after approximately 1000 roughness simulations and is unchanged by further analysis. The size of $\mu$ and associated variance $\sigma$ of those distributions considered in figure 13 are tabulated in table III, while figure 14 illustrates some of the corresponding probability density functions $(\mathrm{PDF})$. 


\begin{tabular}{c|c|c|c}
$N_{2}$ & $x_{\text {len }}$ & $\mu$ & $\sigma$ \\
\hline 80 & 0.02 & 0.000954 & 0.000457 \\
160 & 0.02 & 0.001155 & 0.000580 \\
80 & 0.04 & 0.001066 & 0.000526 \\
160 & 0.04 & 0.001224 & 0.000624 \\
80 & 0.06 & 0.001081 & 0.000542 \\
160 & 0.06 & 0.001213 & 0.000622
\end{tabular}

TABLE III. Mean receptivity amplitudes $\mu$ and corresponding variance $\sigma$ for $\left\{R e_{\infty}, M_{\infty}, \beta\right\}=\left\{5 \times 10^{6}, 0.7,4000\right\}$ and variable short wavelength filter $N_{2}$ and roughness panel length $x_{l e n}$.

(a)
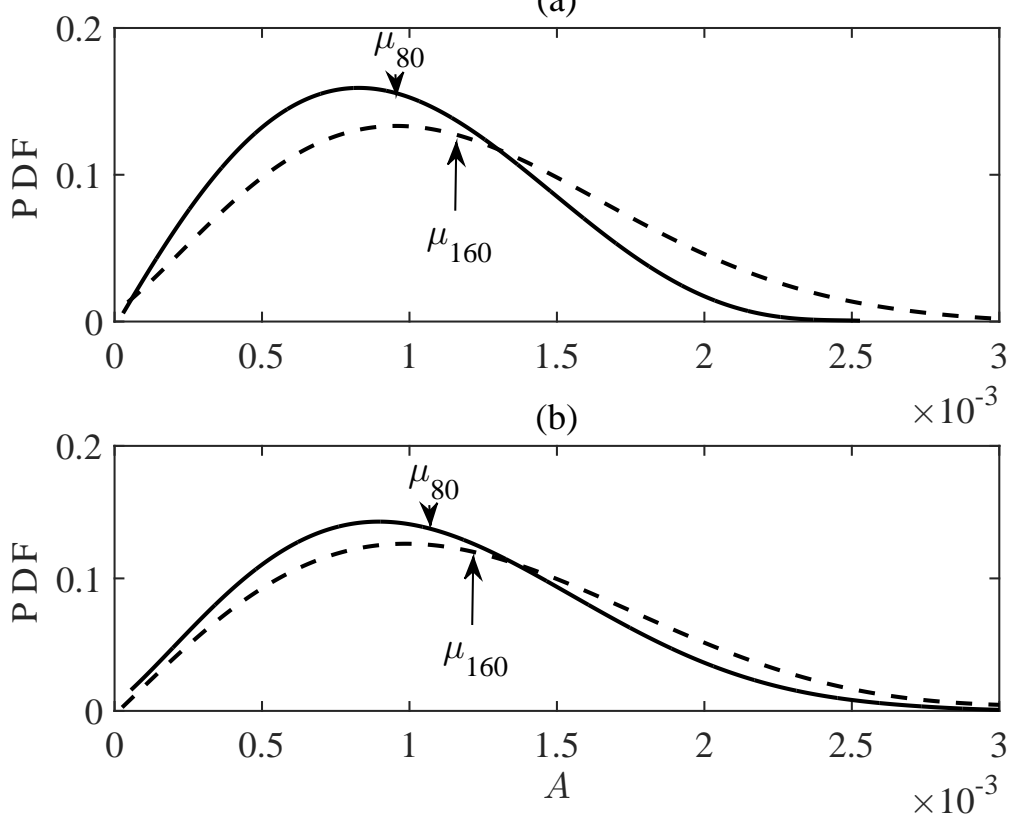

FIG. 14. Probability density functions (PDF) for $\left\{R e_{\infty}, M_{\infty}, \beta\right\}=\left\{5 \times 10^{6}, 0.7,4000\right\}$. (a) $x_{l e n}=0.02$; (b) $x_{l e n}=0.06$. Solid lines illustrate the solution generated for $N_{2}=80$, while dashed lines depict the corresponding result given for $N_{2}=160$.

\section{Variable Roughness Lengths}

The effects of variable roughness length and filter specifications are investigated, where $\mu$ is assumed to have attained a fixed value after $S=5000$ roughness field realisations. Mean amplitudes $\mu$ are plotted in figure 15 against the strip length $x_{l e n}$ for varying short scale wavelength filters $N_{2}$. When $N_{2} \leq 40, \mu$ attains a maximum for $x_{l e n} \approx 0.01$, but for larger strip lengths, $\mu$ is found to decrease and asymptote towards small non-zero values. The reasoning for this particular feature of the receptivity analysis is discussed in greater detail in the subsequent sections. Though essentially it is a direct consequence of the short-scale wavelengths being greater than that associated with the crossflow disturbance. Receptivity is enhanced for roughnesses containing the wavelength of the crossflow instability, which are not included in distributions $N_{2} \leq 40$.

For larger $N_{2}, \mu$ increases and eventually approaches a fixed constant that (although different for each value of $N_{2}$ ) is unchanged by further increments in the length of the roughness strip. This particular finding is consistent with the observations of the adjoint perturbation fields that were strongest about the leading-edge, but diminished for larger $x$. Thus, the effect on receptivity is negligible beyond a fixed chord position, even though the level of surface roughness is not necessarily any less substantial than that found at the leading-edge. 


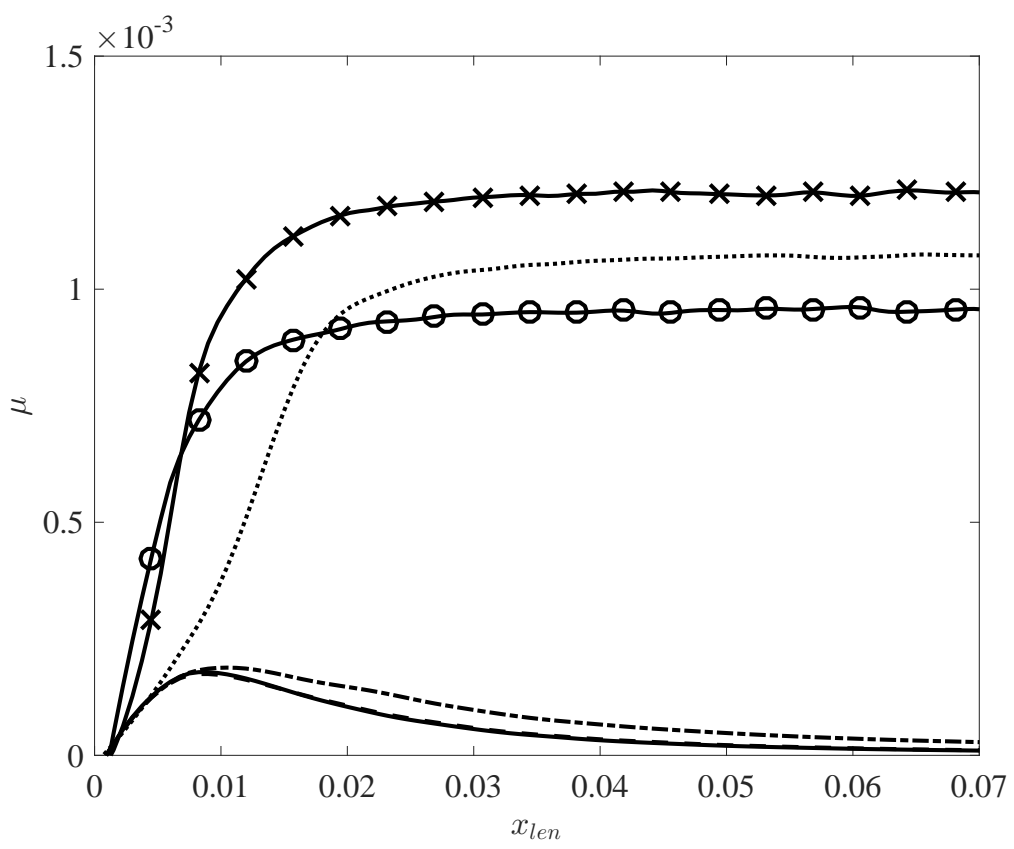

FIG. 15. Mean receptivity amplitude $\mu$ versus length of the roughness panel $x_{\text {len }}$ for $\left\{R e_{\infty}, M_{\infty}, \beta\right\}=\left\{5 \times 10^{6}, 0.7,4000\right\}$, $\lambda_{x}=0.2$ and $N_{2}=10$ (solid line), 20 (dashed), 40 (chain), 80 (dotted), 160 (solid with crosses), 320 (solid with circles).

\section{Short-Scale Filters}

Mean receptivity amplitudes $\mu$ are plotted in figure 16 against $2 \pi N_{2} / \lambda_{x}$ (the wavenumber associated with the shortest scale arising in the cosine series given in (18)), for roughness wavelengths $\lambda_{x}$, lengths $x_{l e n}$ and for increasing streamwise resolution (determined by $N_{x}$-points). The calculations are identical for all three values of $\lambda_{x}$, which is to be expected given the form of the roughness model under consideration; dependant on the ratio $N_{2} / \lambda_{x}$. Additionally, the variation of $\mu$ is qualitatively similar for all $x_{l e n}$ considered, which is again unsurprising given the observations depicted in figure 15 . Furthermore, the magnitude of $\mu$ increases sharply about $2 \pi N_{2} / \lambda_{x} \approx 1200$ or $N_{2} \approx 40$ for $\lambda_{x}=0.2$. The size of $\mu$ is then found to attain a maximum value about $2 \pi N_{2} / \lambda_{x} \approx 4000$, which for $\lambda_{x}=0.2$ corresponds to a short scale wavelength filter within the parameter range $120 \leq N_{2} \leq 160$.

The limiting behaviour of $\mu$ as $2 \pi N_{2} / \lambda_{x}$ tends towards larger values is depicted in figure 16(c), for a variable number of $N_{x}$-points used in the LNS and ALNS streamwise discretisation. For each value of $N_{x}, \mu$ initially decreases and follows the same result. However, the size of $\mu$ is found to deviate around $2 \pi N_{2} / \lambda_{x} \approx\{0.7,1.4,2.8\} \times 10^{5}$ for $N_{x}=2000,4000$ and 8000, respectively. The number of $N_{x}$-points has become insufficient to accurately resolve the very short-scale structures of the roughness distribution. For $N_{x}=16000$ and the range of filters shown, the receptivity amplitude $\mu$ continues to decrease in size and would appear to be tending towards a non-zero constant. Due to computational (RAM memory) limitations, we were unable to extend the calculations for $\mu$ to larger $2 \pi N_{2} / \lambda_{x}$ than that shown in figure 16(c). To ensure that the roughness distribution was accurately discretised for very large $2 \pi N_{2} / \lambda_{x}$, significantly more $N_{x}$-points would have been required. Nevertheless, our calculations suggest that there is a lower limit for $\mu$ as $2 \pi N_{2} / \lambda_{x} \rightarrow \infty$; this of course represents the very fine roughness grain size limit.

In figure 16 the magnitude of $\mu$ was found to increase sharply once the wavenumber $2 \pi N_{2} / \lambda_{x}$ of the shortest roughness scale was greater than a critical value. This particular feature of receptivity to randomly generated surface roughness is investigated further in figure 17. Mean amplitudes $\mu$ are plotted against $2 \pi N_{2} / \lambda_{x}$ for three spanwise wavenumbers, where $\lambda_{x}=0.2$ and $x_{l e n}=0.06$. Eight thousand $\left(N_{x}=8000\right)$ discretised points in the streamwise direction were used to ensure that the roughness distributions were sufficiently resolved, while the freestream conditions were again defined as $\left\{R e_{\infty}, M_{\infty}\right\}=\left\{5 \times 10^{6}, 0.7\right\}$. The three dotted vertical lines in figure 17 represent the value of the wavenumber $\alpha$ associated with each crossflow instability. It is immediately obvious that there is a direct relationship between the size of $\alpha$ and the value of $2 \pi N_{2} / \lambda_{x}$ that is required to establish large receptivity amplitudes. If roughness distributions are only constructed of wavelengths greater than that associated with the crossflow mode, $\mu$ remains relatively small. However, $\mu$ is found to increase by an order of magnitude when the roughness contains shorter scale wavelengths. Thus, in order to maximise the receptivity amplitude to randomly distributed roughness 

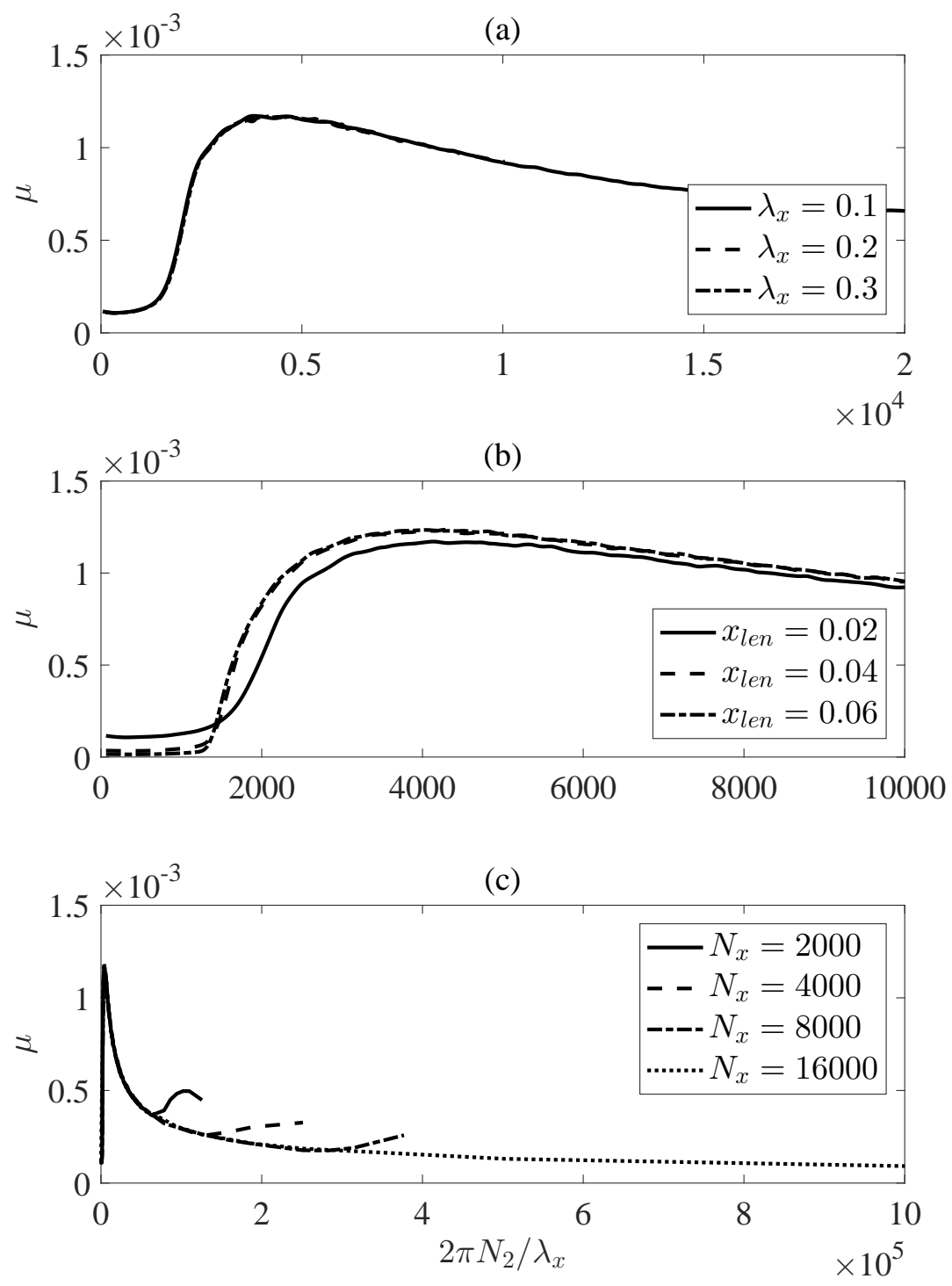

FIG. 16. Mean receptivity amplitude $\mu$ as a function of $2 \pi N_{2} / \lambda_{x}$, for $\left\{R e_{\infty}, M_{\infty}, \beta\right\}=\left\{5 \times 10^{6}, 0.7,4000\right\}$. (a) Number of points $N_{x}=8000, x_{l e n}=0.02$ and variable $\lambda_{x}$. (b) Number of points $N_{x}=8000, \lambda_{x}=0.2$ and variable $x_{l e n}$. (c) $\lambda_{x}=0.2$, $x_{l e n}=0.02$ and variable number of $N_{x}$-points.

of the form (18), the short scale wavelength filter $N_{2}$ must be sufficiently big to ensure that the roughness contains a broad spectrum of wavelengths that includes that associated with the crossflow disturbance wavelength. Conversely, to reduce receptivity, manufacturers must attempt to eliminate the short-scale roughness effects.

\section{Receptivity Control}

The above observations suggest that receptivity may be reduced if the form of the roughness can be controlled. Let us suppose that roughness wavelengths shorter than the crossflow wave can be damped, so that the roughness model 


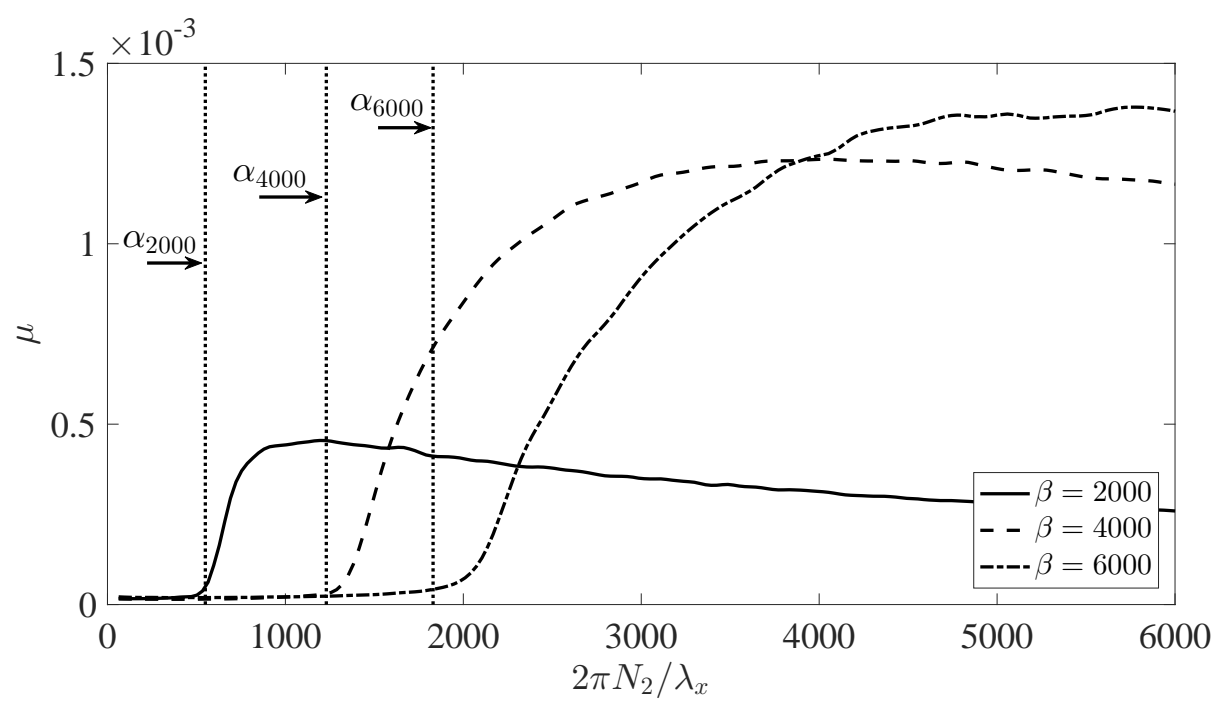

FIG. 17. Mean receptivity amplitude $\mu$ as a function of $2 \pi N_{2} / \lambda_{x}$, for $\beta=2000$ (solid curve), 4000 (dashed), 6000 (chain), and $\left\{R e_{\infty}, M_{\infty}\right\}=\left\{5 \times 10^{6}, 0.7\right\}$. The roughness parameters $\lambda_{x}=0.2, x_{l e n}=0.06$ and the number of points $N_{x}=8000$. The dotted vertical lines represent the wavenumber $\alpha$ associated with the three crossflow modes.

(18) is now of the form

$$
H(x)=\sum_{j=N_{1}}^{N_{2}} \mathcal{A} a_{j} \cos \left(2 \pi\left[j x / \lambda_{x}+\phi_{j}\right]\right),
$$

where $\mathcal{A}$ acts as a scale mechanism for the short wavelength structures

$$
\mathcal{A}=1 \quad \text { for } \quad j \leq N_{\alpha} \text { and } \mathcal{A}=\epsilon<1 \text { for } j>N_{\alpha},
$$

where $N_{\alpha}$ is the value of the filter that establishes a wavelength equal to that given for the crossflow instability. For $\beta=4000$ and roughness distributions given by $\lambda_{x}=0.2$ and $x_{l e n}=0.06$, filters $N_{\alpha} \approx 40$ are found to establish wavelengths equal to that corresponding to the crossflow disturbance. Thus, in order to successfully dampen the receptivity amplitude, all waves $j>40$ are scaled on $\epsilon$.

Figure 18(a) displays the $\mu$-calculations against $2 \pi N_{2} / \lambda_{x}$ for several scale factors $\epsilon$. Suppressing the short wavelength roughness scales establishes a reduction in the mean amplitude, with maxima again obtained for $2 \pi N_{2} / \lambda_{x}>$ 4000 (about $N_{2}=160$ for $\lambda_{x}=0.2$ ). In 18(b), $\mu$ is plotted against $\epsilon$ for $N_{2}=160$. Cross markers are used to represent actual calculations, while the solid curve was constructed using a spline fitting. The dotted horizontal line denotes the size of $\mu$ obtained in the limit $\epsilon=0$. Amplitudes are respectively reduced to $1 / 5$ th and $1 / 30$ th of their original size, for $\epsilon=0.1$ and 0.01 . (Note that the receptivity amplitude equates to about $1 / 38$ th when $\epsilon=0$ ). Thus, a reasonable reduction in the receptivity amplitude can be established if the short scale wavelengths are suppressed.

\section{Mean Amplitude for Variable $\beta$ and $R e_{\infty}$}

For the remaining analysis, the roughness parameters $\lambda_{x}$ and $N_{2}$ are respectively set to 0.2 and 160 . These particular specifications are chosen to ensure that the roughness distributions are constructed of many wavelengths, including that associated with the crossflow instability. A contour of $\mu$ is plotted in figure 19 for variable $\beta$ and $R e_{\infty}$. The results correspond to roughness distributions with a fixed length $x_{l e n}=0.06$ and a Mach number $M_{\infty}=0.7$. A maximum amplitude is located about $R e_{\infty}=5 \times 10^{6}$ and $\beta \approx 5000$, which would appear to contradict the earlier results illustrated in figure 11 where the two adjoint perturbation fields $\left|u_{y, y=0}^{*}\right| / R e$ and $\left|w_{y, y=0}^{*}\right| / R e$ (required for predicting receptivity) established greater maxima for larger $R e_{\infty}$. Nevertheless, a closer examination of the two adjoint perturbation fields (albeit difficult to identify in the illustrations drawn in figure 11) indicate that $\left|u_{y, y=0}^{*}\right| / R e$ and $\left|w_{y, y=0}^{*}\right| / R e$ remain non-negligible for a greater chord length for the smaller $R e_{\infty}$ calculations. Hence, we obtain the results shown in figure 19. 


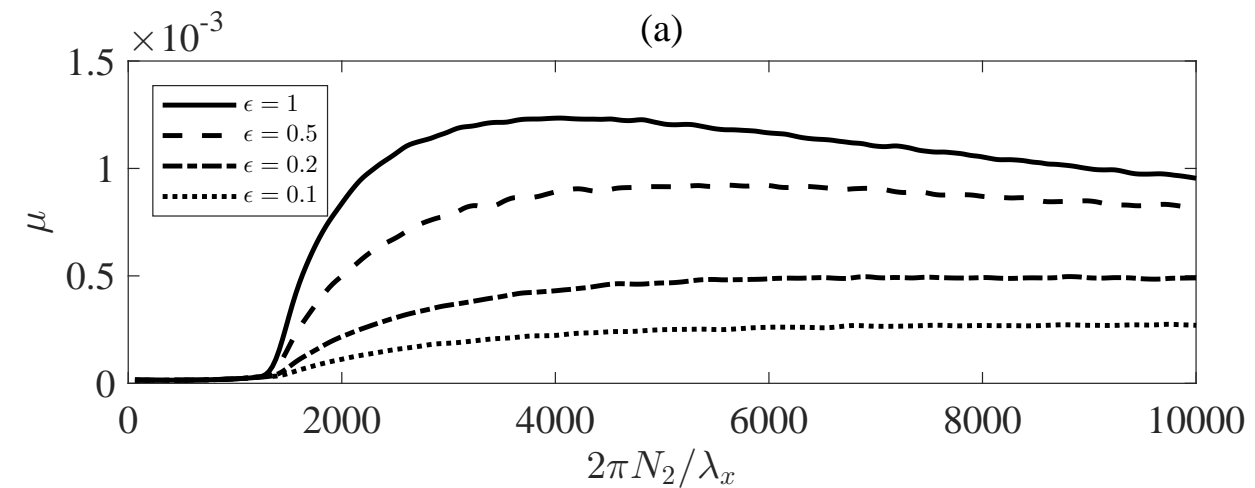

(b)

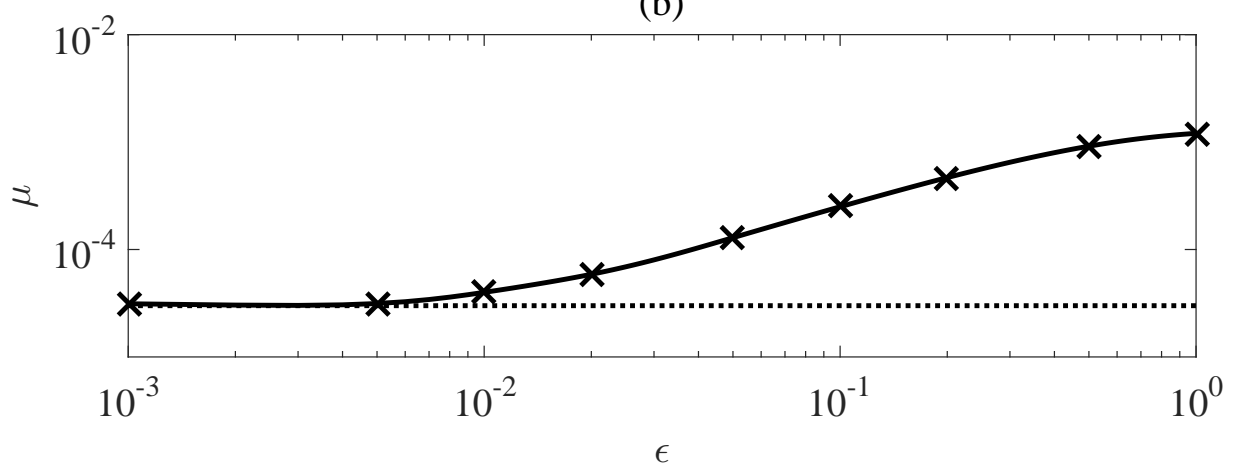

FIG. 18. Mean receptivity amplitude as a function of (a) $2 \pi N_{2} / \lambda_{x}$ and variable $\epsilon$; (b) $\epsilon$ and $N_{2}=160$ for $\lambda_{x}=0.2, x_{l e n}=0.06$ and $\beta=6000$.

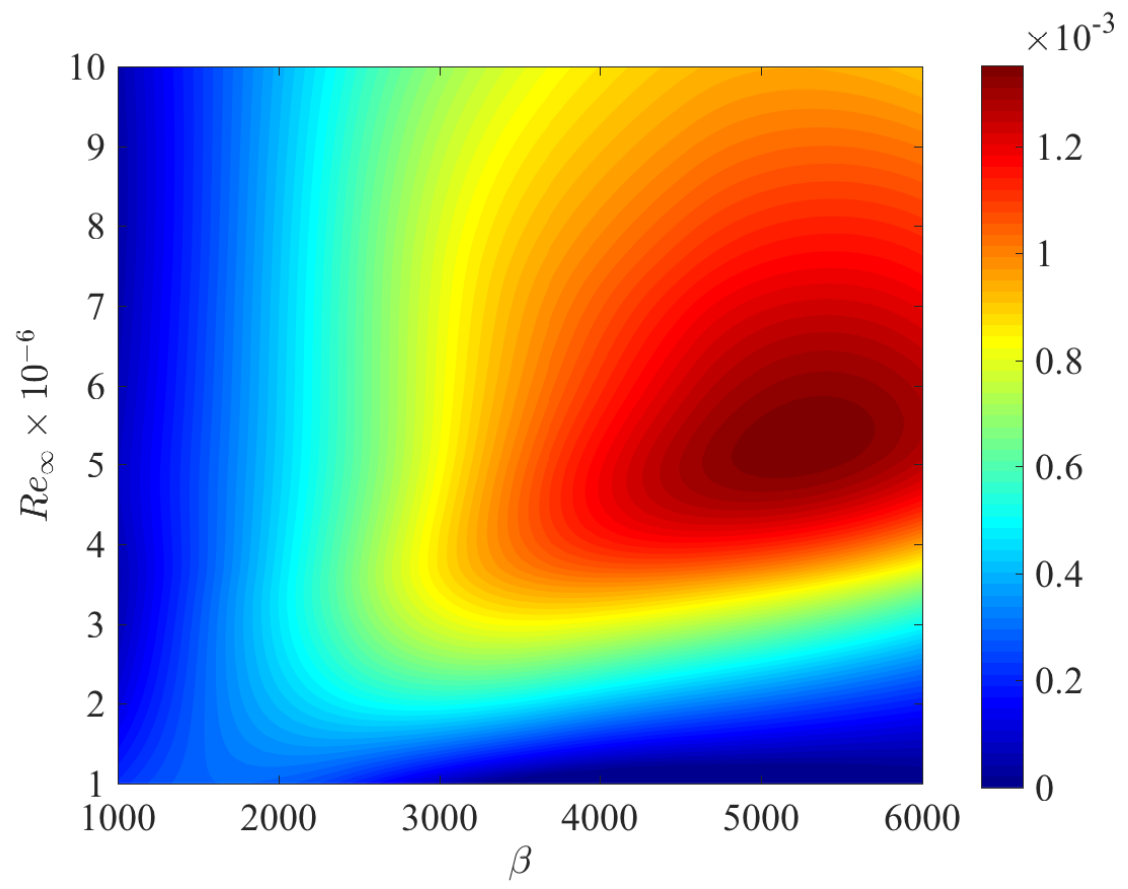

FIG. 19. Contours depicting the mean receptivity amplitude $\mu$ in the $\left\{\beta, R e_{\infty}\right\}$-plane for $M_{\infty}=0.7, N_{2}=160$ and $x_{l e n}=0.06$. 


\section{CONCLUSIONS}

Receptivity of stationary crossflow disturbances on an infinite swept wing have been investigated using solutions of the ALNS equations. The adjoint method for predicting the amplitude of perturbations to variations in the wing surface roughness was described, and applied to both incompressible and compressible flow LNS formulations. Analysis was undertaken for a broad spectrum of spanwise wavenumbers, and several freestream Reynolds numbers and subsonic Mach numbers. Crossflow disturbances were most affected by surface roughness variations located close to the leading-edge of the swept wing, while the effects of curvature were found to feature prominently in the receptivity calculations. These observations are consistent with the results of previous receptivity studies performed on different wing geometries ${ }^{19}$. Additionally, the conclusions regarding receptivity being strongest about the leading-edge, reflect experimental observations ${ }^{16}$. For the model considered, compressible flow effects were found to be negligible in the receptivity calculations. However, compressibility was significant in disturbance growth rates and hence amplitude evolution further downstream. Furthermore, as our analysis was limited to subsonic flight conditions $\left(M_{\infty} \leq 0.7\right)$, compressibility may still play a significant role in receptivity predictions in supersonic regimes.

As the adjoint based receptivity method only requires that the LNS and ALNS systems be solved once, we were able to undertake a fast and efficient Monte-Carlo type analysis involving many thousands of randomly generated roughness permutations. A 2D randomly distributed roughness model was implemented and used to conduct an extensive study of linear receptivity characteristics. Receptivity amplitudes to roughness strips could be computed instantaneously using the adjoint formulation, overcoming the huge computational and time costs associated with undertaking a comparable LNS study. An extensive investigation was carried out for roughness distributions of variable heights, wavelengths and filter specifications. Mean disturbance amplitudes and variances were computed for a range of flow conditions and roughness parameter settings, by averaging over many receptivity calculations. The predicted mean receptivity amplitudes were found to approach a constant value for sufficiently long roughness strips, which was unchanged by further increases in the roughness length. For the given roughness model, receptivity was strongest for roughness distributions containing a broad spectrum of wavelengths, which included that associated with the dominant crossflow mode. Additionally, it was shown that by damping the very short roughness scales, a reduction in the generated crossflow amplitude is achieved. Hence, manufacturers of wing surfaces may be able to delay stationary crossflow induced transition by suppressing the short scale structures.

Subsequent stages of the receptivity processes to randomly distributed roughness, including nonlinearity and secondary instabilities, might then be investigated using the the predictions of the Monte-Carlo analysis. The mean amplitudes derived from the linear receptivity analysis can be used to force nonlinear PSE simulations. Utilising the variances, allows bounds on the expected transition location to be determined.

Our primary interest in this study has been to demonstrate the fast and efficient adjoint based method for predicting linear receptivity to randomly distributed surface roughness. Although roughness plays a significant role in the laminar-turbulent transition process (particularly about the wings leading-edge) we should acknowledge that unstable travelling crossflow modes may be generated by the environmental conditions. Additionally, the effects of freestream turbulence and 3D mechanisms should be included in a more complete study of receptivity.

This work has been supported by the EPSRC funded LFC-UK project: Development of Under-pinning Technology for Laminar Flow Control, grant EP/I037946/1 and by the Innovate UK funded ALFET project 113022. We thank the referees for many helpful suggestions concerning the presentation and improvement of our results.

\section{Appendix A: Incompressible Flow Formulation}

\section{Linearised Navier-Stokes Equations}

The full LNS equations for an incompressible flow $\mathbf{Q}_{\mathbf{B}}(x, y)=\left\{P_{B}, \mathbf{U}_{\mathbf{B}}\right\}(x, y)$ with linear perturbations $\overline{\mathbf{q}}$ of the form (1a) are given as

$$
\begin{gathered}
\chi u_{x}+v_{y}-\kappa \chi v+i b w=0, \\
\frac{u_{y y}+\chi^{2} u_{x x}}{R e}-\left\{\mathcal{B}_{1}+\frac{\kappa^{2} \chi^{2}}{R}+\chi\left(U_{B, x}-\kappa V_{B}\right)\right\} u-\chi p_{x}-\mathcal{B}_{2} u_{y}+\left\{\kappa \chi U_{B}-U_{B, y}\right\} v-\chi U_{B} u_{x}-\frac{2 \kappa \chi^{2}}{R e} v_{x}=0, \\
\frac{v_{y y}+\chi^{2} v_{x x}}{R e}-\left\{\mathcal{B}_{1}+\frac{\kappa^{2} \chi^{2}}{R e}+V_{B, y}\right\} v-p_{y}-\mathcal{B}_{2} v_{y}-\left\{2 \kappa U_{B}+V_{B, x}\right\} \chi u-\chi U_{B} v_{x}+\frac{2 \kappa \chi^{2}}{R e} u_{x}=0,
\end{gathered}
$$




$$
\frac{w_{y y}+\chi^{2} w_{x x}}{R e}-\mathcal{B}_{1} w-i b p-\chi W_{B, x} u-W_{B, y} v-\mathcal{B}_{2} w_{y}-\chi U_{B} w_{x}=0
$$

where

$$
\mathcal{B}_{1}=i\left(b W_{B}-\omega\right)+\frac{b^{2}}{R e} \quad \text { and } \quad \mathcal{B}_{2}=V_{B}+\frac{\kappa \chi}{R e}
$$

while $\kappa$ and $\chi$ are the body surface curvature terms that are assumed (for simplicity) to be independent of the chord $x$-direction. Here $\kappa$ is the local body curvature and

$$
\chi=\frac{1}{1-\kappa y} .
$$

Thus, in the large curvature limit, $\kappa=0$ and $\chi=1$.

\section{Adjoint Linearised Navier-Stokes Equations}

The corresponding ALNS system of equations are then derived and given as

$$
\begin{gathered}
\chi u_{x}^{*}+v_{y}^{*}-i b w^{*}=0 \\
\frac{u_{y y}^{*}+\chi^{2} u_{x x}^{*}}{R e}-\left\{\mathcal{B}_{1}+\chi\left(U_{B, x}-2 \kappa V_{B}\right)\right\} u^{*}+\chi p_{x}^{*}+\mathcal{B}_{2} u_{y}^{*}-\chi\left(V_{B, x}+2 \kappa U_{B}\right) v^{*}+\chi U_{B} u_{x}^{*}-\frac{2 \kappa \chi^{2}}{R e} v_{x}^{*}-\chi W_{B, x} w^{*}=0, \\
\frac{v_{y y}^{*}+\chi^{2} v_{x x}^{*}}{R e}-\left\{\mathcal{B}_{1}+V_{B, y}-\kappa \chi V_{B}\right\} v^{*}+p_{y}^{*}+\kappa \chi p^{*}+\mathcal{B}_{2} v_{y}^{*}-\left\{U_{B, y}-\kappa \chi U_{B}\right\} u^{*}+\chi U_{B} v_{x}^{*}+\frac{2 \kappa \chi^{2}}{R e} u_{x}^{*}-W_{B, y} w^{*}=0, \\
\frac{w_{y y}^{*}+\chi^{2} w_{x x}^{*}}{R e}-\left\{\mathcal{B}_{1}-\frac{\kappa^{2} \chi^{2}}{R e}-\kappa \chi V_{B}\right\} w^{*}-i b p^{*}+\mathcal{B}_{2} w_{y}^{*}+\chi U_{B} w_{x}^{*}=0,
\end{gathered}
$$

where we have assumed that adjoint perturbations are of the form (7).

\section{K-Operator}

The bi-linear concomitant $\mathbf{K}=\left\{K_{t}, K_{x}, K_{y}, K_{z}\right\}$-operator that is associated with the above LNS and ALNS systems, is defined as

$$
\begin{aligned}
& K_{t}=\mathbf{u} \cdot \mathbf{u}^{*} \\
& K_{x}=\chi U_{B} \mathbf{u} \cdot \mathbf{u}^{*}+\chi\left(u p^{*}+p u^{*}\right)+\frac{\chi^{2}}{R e}\left(\mathbf{u} \cdot \mathbf{u}_{x}^{*}-\mathbf{u}_{x} \cdot \mathbf{u}^{*}\right)+\frac{2 \kappa \chi^{2}}{R e}\left(v u^{*}-u v^{*}\right), \\
& K_{y}=\mathcal{B}_{2} \mathbf{u} \cdot \mathbf{u}^{*}+v p^{*}+p v^{*}+\frac{1}{R e}\left(\mathbf{u} \cdot \mathbf{u}_{y}^{*}-\mathbf{u}_{y} \cdot \mathbf{u}^{*}\right), \\
& K_{z}=W_{B} \mathbf{u} \cdot \mathbf{u}^{*}+w p^{*}+p w^{*}-\frac{2 i b}{R e}\left(\mathbf{u} \cdot \mathbf{u}^{*}\right)
\end{aligned}
$$

where

$$
\mathbf{u} \cdot \mathbf{u}^{*}=u u^{*}+v v^{*}+w w^{*} \quad \text { and } \quad \mathbf{u}_{x}=\left\{u_{x}, v_{x}, w_{x}\right\} \text {. }
$$




\section{Appendix B: Compressible Flow Formulation}

\section{Linearised Navier-Stokes Equations}

The body fitted LNS system of equations for a compressible flow $\mathbf{Q}_{\mathbf{B}}=\left\{P_{B}, U_{B}, V_{B}, W_{B}, T_{B}\right\}$ and linear perturbations

$$
\overline{\mathbf{q}}(\mathbf{x}, t)=\{p, u, v, w, T\}(x, y) \exp \{i(b z-\omega t)\}
$$

are defined as:

$$
\begin{aligned}
& \chi u_{x}+\frac{\chi \rho_{B, x}}{\rho_{B}} u+\left\{\frac{\rho_{B, y}}{\rho_{B}}-\kappa \chi\right\} v+v_{y}+i b w+\frac{\mathcal{C}_{1}}{P_{B}} p+\frac{\chi U_{B} p_{x}+V_{B} p_{y}}{P_{B}}-\frac{\left(\chi U_{B} T_{x}+V_{B} T_{y}\right)}{T_{B}}-\left\{\mathcal{C}_{1}+\mathcal{C}_{5}\right\} \frac{T}{T_{B}}=0 \\
& \frac{u_{y y}+r \chi^{2} u_{x x}}{R e}+\frac{s \chi}{R e}\left\{v_{x y}+i b w_{x}\right\}+\frac{\mathcal{E}_{1}}{P_{B}} p-\frac{\chi}{\mu_{B}} p_{x}-\left\{\frac{\chi \rho_{B}}{\mu_{B}}\left(U_{B, x}-\kappa V_{B}\right)+\mathcal{C}_{3}+\frac{\kappa \chi}{R e}\left(\kappa \chi-\mathcal{F}_{3}\right)\right\} u-\mathcal{F}_{1} u_{y}- \\
& \left\{\mathcal{F}_{2}-\frac{s \chi \mathcal{F}_{4}}{R e}\right\} u_{x}+\frac{m \mathcal{F}_{4}}{R e} v_{y}-\frac{\chi}{R e}\left\{e \kappa \chi-\mathcal{F}_{3}\right\} v_{x}+\left\{\frac{\rho_{B}}{\mu_{B}}\left(\kappa \chi U_{B}-U_{B, y}\right)-\frac{r \kappa \chi \mathcal{F}_{4}}{R e}\right\} v+\frac{i b m \mathcal{F}_{4}}{R e} w+ \\
& \frac{f_{T}}{\mu_{B} R e}\left\{\chi \mathcal{D}_{2} T_{x}+\mathcal{D}_{1} T_{y}\right\}+\left\{\frac{f_{T T}}{\mu_{B} R e}\left(\chi \mathcal{D}_{2} T_{B, x}+\mathcal{D}_{1} T_{B, y}\right)-\frac{\mathcal{E}_{1}}{T_{B}}+\frac{f_{T}}{\mu_{B} R e}\left(i b \chi W_{B, x}+\mathcal{G}_{1}-\kappa \chi \mathcal{D}_{1}\right)\right\} T=0 \\
& \frac{r v_{y y}+\chi^{2} v_{x x}}{R e}+\frac{s}{R e}\left\{\chi u_{x y}+i b w_{y}\right\}-\frac{\mathcal{E}_{2}}{P_{B}} p-\frac{p_{y}}{\mu_{B}}-\left\{\frac{\rho_{B} V_{B, y}}{\mu_{B}}+\mathcal{C}_{3}+\frac{\kappa \chi}{R e}\left(r \kappa \chi+m \mathcal{F}_{3}\right)\right\} v-\left\{\mathcal{F}_{1}+\frac{s}{R e}\left(\kappa \chi-\mathcal{F}_{3}\right)\right\} v_{y}- \\
& \mathcal{F}_{2} v_{x}+\frac{\mathcal{F}_{4}}{R e} u_{y}+\frac{\chi}{R e}\left\{e \kappa \chi+m \mathcal{F}_{3}\right\} u_{x}-\left\{\frac{\rho_{B}}{\mu_{B}}\left(2 \kappa U_{B}+V_{B, x}\right)-\frac{\kappa \mathcal{F}_{4}}{R e}\right\} \chi u+\frac{i b m \mathcal{F}_{3}}{R e} w+ \\
& \frac{f_{T}}{\mu_{B} R e}\left\{\chi \mathcal{D}_{1} T_{x}+\mathcal{D}_{3} T_{y}\right\}+\left\{\frac{f_{T T}}{\mu_{B} R e}\left(\chi \mathcal{D}_{1} T_{B, x}+\mathcal{D}_{3} T_{B, y}\right)+\frac{\mathcal{E}_{2}}{T_{B}}+\frac{f_{T}}{\mu_{B} R e}\left(i b W_{B, y}+\mathcal{G}_{2}-r \kappa \chi \mathcal{D}_{5}\right)\right\} T=0 \\
& \frac{w_{y y}+\chi^{2} w_{x x}}{R e}+\frac{i b s}{R e}\left\{\chi u_{x}+v_{y}\right\}-\left\{\frac{i b}{\mu_{B}}+\frac{\mathcal{E}_{3}}{P_{B}}\right\} p-\mathcal{F}_{1} w_{y}-\mathcal{F}_{2} w_{x}-\left\{\mathcal{C}_{3}+\frac{s b^{2}}{R e}\right\} w- \\
& \left\{\frac{\rho_{B} \chi W_{B, x}}{\mu_{B}}-\frac{i b \mathcal{F}_{4}}{R e}\right\} u-\left\{\frac{\rho_{B} W_{B, y}}{\mu_{B}}+\frac{i b}{R e}\left(s \kappa \chi-\mathcal{F}_{3}\right)\right\} v+\frac{f_{T}}{\mu_{B} R e}\left\{\chi^{2} W_{B, x} T_{x}+W_{B, y} T_{y}\right\}+ \\
& \left\{\frac{f_{T T}}{\mu_{B} R e}\left(\chi^{2} W_{B, x} T_{B, x}+W_{B, y} T_{B, y}\right)+\frac{\mathcal{E}_{3}}{T_{B}}+\frac{f_{T}}{\mu_{B} R e}\left(i b m \mathcal{D}_{4}-\kappa \chi W_{B, y}+\mathcal{G}_{3}\right)\right\} T=0 \\
& \frac{T_{y y}+\chi^{2} T_{x x}}{R e}+\frac{\Gamma}{\mu_{B}}\left\{\chi U_{B} p_{x}+V_{B} p_{y}\right\}+\left\{\frac{\Gamma \mathcal{C}_{2}}{\mu_{B}}-\frac{\sigma \mathcal{E}_{4}}{P_{B}}\right\} p+\frac{\chi}{R e}\left\{\mathcal{H}_{3}+\mathcal{F}_{4}\right\} T_{x}+\left\{\mathcal{H}_{4}+\mathcal{F}_{3}\right\} \frac{T_{y}}{R e}+ \\
& \left\{\mathcal{H}_{1}+\frac{2 \Gamma}{R e}\left(\kappa \chi \mathcal{D}_{1}+i b \chi W_{B, x}\right)\right\} u+\left\{\mathcal{H}_{2}+\frac{2 \Gamma}{R e}\left(i b W_{B, y}-\kappa \chi \mathcal{D}_{3}\right)\right\} v+\frac{2 \Gamma}{R e}\left\{\mathcal{D}_{1} u_{y}+\mathcal{D}_{3} v_{y}+W_{B, y} w_{y}+\right. \\
& \left.\chi \mathcal{D}_{2} u_{x}+\chi \mathcal{D}_{1} v_{x}+\chi^{2} W_{B, x} w_{x}+i m \beta \mathcal{D}_{6} w\right\}+\left\{\frac{\sigma \mathcal{E}_{4}}{T_{B}}-\mathcal{C}_{4}+\frac{f_{T T}}{\mu_{B} R e}\left(\chi^{2} T_{B, x}^{2}+T_{B, y}^{2}\right)+\frac{f_{T}}{\mu_{B} R e}\left(\mathcal{G}_{4}+\mathcal{G}_{5}\right)\right\} T^{T}=0
\end{aligned}
$$

\section{Adjoint Linearised Navier-Stokes Equations}

The corresponding ALNS system of equations for

$$
\overline{\mathbf{q}}^{*}(\mathbf{x}, t)=\left\{p^{*}, u^{*}, v^{*}, w^{*}, T^{*}\right\}(x, y) \exp \{i(\omega t-b z)\}
$$


are given as:

$$
\begin{aligned}
& \frac{\mu_{B} \mathcal{E}_{1}}{P_{B}} u^{*}+\chi u_{x}^{*}-\frac{\mu_{B} \mathcal{E}_{2}}{P_{B}} v^{*}+v_{y}^{*}-\frac{\mu_{B} \mathcal{E}_{3}}{P_{B}} w^{*}-\left\{\frac{\sigma \mu_{B} \mathcal{E}_{4}}{P_{B}}+\Gamma\left(\chi U_{B, x}+V_{B, y}-\mathcal{C}_{2}\right)\right\} T^{*}+\left\{\kappa \chi V_{B}-\mathcal{C}_{2}-\mathcal{C}_{5}\right\} \frac{p^{*}}{P_{B}}+ \\
& \left\{\frac{\chi U_{B} p_{x}^{*}+V_{B} p_{y}^{*}}{P_{B}}\right\}-\Gamma\left\{\chi U_{B} T_{x}^{*}+V_{B} T_{y}^{*}\right\}=0 \\
& \frac{u_{y y}^{*}+r \chi^{2} u_{x x}^{*}}{R e}+\frac{s \chi}{R}\left\{v_{x y}^{*}-i b w_{x}^{*}\right\}+\frac{\chi}{\mu_{B}}\left\{p_{x}^{*}-\frac{\rho_{B, x}}{\rho_{B}} p^{*}\right\}-\left\{\frac{\chi \rho_{B}}{\mu_{B}}\left(U_{B, x}-2 \kappa V_{B}\right)+\mathcal{C}_{3}-\frac{2 \kappa \chi \mathcal{F}_{3}}{R}\right\} u^{*}+\mathcal{F}_{1} u_{y}^{*}+ \\
& \left\{\mathcal{F}_{2}+\frac{s \chi \mathcal{F}_{4}}{R e}\right\} u_{x}^{*}-\left\{\frac{\rho_{B}}{\mu_{B}}\left(2 \kappa U_{B}+V_{B, x}\right)+\frac{2 \kappa \mathcal{F}_{4}}{R e}\right\} \chi v^{*}+\frac{m \mathcal{F}_{4}}{R e} v_{y}^{*}-\frac{\chi}{R e}\left\{2 \kappa \chi-\mathcal{F}_{3}\right\} v_{x}^{*}-\left\{\frac{\chi \rho_{B} W_{B, x}}{\mu_{B}}+\frac{i b m \mathcal{F}_{4}}{R e}\right\} w^{*}- \\
& \frac{2 \Gamma}{R e}\left\{\chi \mathcal{D}_{2} T_{x}^{*}+\mathcal{D}_{1} T_{y}^{*}\right\}+\left\{\mathcal{H}_{1}+\frac{2 \Gamma}{R e}\left(i b \chi W_{B, x}-\mathcal{G}_{1}-\mathcal{D}_{2} \mathcal{F}_{4}-\mathcal{D}_{1} \mathcal{F}_{3}\right)\right\} T^{*}=0 \\
& \frac{r v_{y y}^{*}+\chi^{2} v_{x x}^{*}}{R e}+\frac{s}{R}\left\{\chi u_{x y}^{*}-i b w_{y}^{*}\right\}+\left\{\frac{p_{y}^{*}}{\mu_{B}}+\left(\kappa \chi-\frac{\rho_{B, y}}{\rho_{B}}\right) \frac{p^{*}}{\mu_{B}}\right\}-\left\{\frac{\rho_{B}}{\mu_{B}}\left(V_{B, y}-\kappa \chi V_{B}\right)+\mathcal{C}_{3}-\frac{2 \kappa \chi \mathcal{F}_{3}}{R e}\right\} v^{*}+\left\{\mathcal{F}_{1}+\frac{s \mathcal{F}_{3}}{R e}\right\} v_{y}^{*}+ \\
& \mathcal{F}_{2} v_{x}^{*}-\left\{\frac{\rho_{B}}{\mu_{B}}\left(U_{B, y}-\kappa \chi U_{B}\right)-\frac{2 \kappa \chi \mathcal{F}_{4}}{R e}\right\} u^{*}+\frac{\mathcal{F}_{4}}{R} u_{y}^{*}+\frac{\chi}{R e}\left\{2 r \kappa \chi+m \mathcal{F}_{3}\right\} u_{x}^{*}-\left\{\frac{\rho_{B} W_{B, y}}{\mu_{B}}+\frac{i b}{R e}\left(m \mathcal{F}_{3}+s \kappa \chi\right)\right\} w^{*}- \\
& \frac{2 \Gamma}{R e}\left\{\chi \mathcal{D}_{1} T_{x}^{*}+\mathcal{D}_{3} T_{y}^{*}\right\}+\left\{\mathcal{H}_{2}+\frac{2 \Gamma}{R e}\left(i b W_{B, y}+s \kappa \chi\left(\mathcal{D}_{5}-\mathcal{D}_{4}\right)-\mathcal{G}_{2}-\mathcal{D}_{1} \mathcal{F}_{3}-\mathcal{D}_{3} \mathcal{F}_{4}\right)\right\} T^{*}=0 \\
& \frac{w_{y y}^{*}+\chi^{2} w_{x x}^{*}}{R e}-\frac{i \beta s}{R e}\left\{\chi u_{x}^{*}+v_{y}^{*}\right\}-\frac{i b}{\mu_{B}} p^{*}+\left\{\frac{\kappa \chi \rho_{B} V_{B}}{\mu_{B}}-\mathcal{C}_{3}+\frac{\kappa^{2} \chi^{2}-s b^{2}}{R e}+\frac{\kappa \chi \mathcal{F}_{3}}{R e}\right\} w^{*}+\mathcal{F}_{2} w_{x}^{*}+\mathcal{F}_{1} w_{y}^{*}- \\
& \frac{i b \mathcal{F}_{4}}{R e} u^{*}-\frac{i b \mathcal{F}_{3}}{R e} v^{*}-\frac{2 \Gamma}{R e}\left\{\chi^{2} W_{B, x} T_{x}^{*}+W_{B, y} T_{y}^{*}\right\}+\frac{2 \Gamma}{R e}\left\{i b m \mathcal{D}_{4}-\chi \mathcal{F}_{4} W_{B, x}-\mathcal{F}_{3} W_{B, y}-\mathcal{G}_{3}\right\} T^{*}=0 \\
& \frac{T_{y y}^{*}+\chi^{2} T_{x x}^{*}}{R e}+\left\{\mathcal{C}_{2}+\mathcal{C}_{5}-\kappa \chi V_{B}\right\} \frac{p^{*}}{\mu_{B} T_{B}}-\frac{\left(\chi U_{B} p_{x}^{*}+V_{B} p_{y}^{*}\right)}{\mu_{B} T_{B}}-\frac{\chi}{R e}\left\{\mathcal{H}_{3}-\mathcal{F}_{4}\right\} T_{x}^{*}-\left\{\mathcal{H}_{4}-\mathcal{F}_{3}\right\} \frac{T_{y}^{*}}{R e}- \\
& \frac{f_{T}}{\mu_{B} R e}\left\{\chi \mathcal{D}_{2} u_{x}^{*}+\chi \mathcal{D}_{1} v_{x}^{*}+\chi^{2} W_{B, x} w_{x}^{*}+\mathcal{D}_{1} u_{y}^{*}+\mathcal{D}_{3} v_{y}^{*}+W_{B, y} w_{y}^{*}\right\}+\left\{\frac{\mathcal{E}_{1}}{T_{B}}+\frac{f_{T}}{\mu_{B} R e}\left(2 \kappa \chi \mathcal{D}_{1}-i b \chi W_{B, x}\right)\right\} u^{*}+ \\
& \left\{\frac{\mathcal{E}_{2}}{T_{B}}+\frac{f_{T}}{\mu_{B} R e}\left(2 \kappa \chi \mathcal{D}_{4}+i b W_{B, y}\right)\right\} v^{*}+\left\{\frac{\mathcal{E}_{3}}{T_{B}}+\frac{i b m f_{T}}{\mu_{B} R e} \mathcal{D}_{4}\right\} w^{*}-\left\{\sigma\left(\frac{\mathcal{E}_{4}}{T_{B}}+\frac{\kappa \chi \rho_{B} V_{B}}{\mu_{B}}\right)-\mathcal{C}_{4}+\frac{\kappa^{2} \chi^{2}}{R e}+\frac{f_{T} \mathcal{G}_{5}}{\mu_{B} R e}\right\} T^{*}=0
\end{aligned}
$$

The parameters $\Gamma=(\gamma-1) M_{\infty}^{2} \sigma, e=r+1, r=s+1, s=m+1$ and $m=-2 / 3=\lambda / \mu$. (The Stokes relation is used to define the second coefficient of viscosity $\lambda$ ). Here $\mu_{B}=f(T)$ is the dependence of the dynamic viscosity on the temperature (Sutherland's relationship) and $f_{T}=d \mu_{B} / d T, f_{T T}=d^{2} \mu_{B} / d T^{2}$. Further, $\sigma$ represents the Prandtl 
number, $\gamma$ is the ratio of the specific heats and $M_{\infty}$ is the Mach number. The terms $\mathcal{C}_{1}, \mathcal{C}_{2}, \ldots, \mathcal{H}_{4}$ are defined as

$$
\begin{aligned}
& \mathcal{C}_{1}=i\left(b W_{B}-\omega\right)+\chi\left(U_{B, x}-\kappa V_{B}\right)+V_{B, y}-\frac{\left(\chi U_{B} T_{B, x}+V_{B} T_{B, y}\right)}{T_{B}}, \\
& \mathcal{C}_{2}=i\left(b W_{B}-\omega\right) \\
& \mathcal{C}_{3}=\frac{i \rho_{B}}{\mu_{B}}\left(\beta W_{B}-\omega\right)+\frac{b^{2}}{R e} \\
& \mathcal{C}_{4}=\frac{i \sigma \rho_{B}}{\mu_{B}}\left(\beta W_{B}-\omega\right)+\frac{b^{2}}{R e}, \\
& \mathcal{C}_{5}=\frac{\chi \rho_{B, x} U_{B}+\rho_{B, y} V_{B}}{\rho_{B}}, \\
& \mathcal{D}_{1}=U_{B, y}+\chi\left(V_{B, x}+\kappa U_{B}\right), \\
& \mathcal{D}_{2}=m V_{B, y}+r \chi\left(U_{B, x}-\kappa V_{B}\right), \\
& \mathcal{D}_{3}=r V_{B, y}+m \chi\left(U_{B, x}-\kappa V_{B}\right), \\
& \mathcal{D}_{4}=V_{B, y}+\chi\left(U_{B, x}-\kappa V_{B}\right) \text {, } \\
& \mathcal{D}_{5}=V_{B, y}-\chi\left(U_{B, x}-\kappa V_{B}\right) \text {, } \\
& \mathcal{E}_{1}=\frac{\rho_{B}}{\mu_{B}}\left\{\chi U_{B}\left(\kappa V_{B}-U_{B, x}\right)-V_{B} U_{B, y}\right\}, \\
& \mathcal{E}_{2}=\frac{\rho_{B}}{\mu_{B}}\left\{\chi U_{B}\left(\kappa U_{B}+V_{B, x}\right)+V_{B} V_{B, y}\right\}, \\
& \mathcal{E}_{3}=\frac{\rho_{B}}{\mu_{B}}\left\{\chi U_{B} W_{B, x}+V_{B} W_{B, y}\right\}, \\
& \mathcal{E}_{4}=\frac{\rho_{B}}{\mu_{B}}\left\{\chi U_{B} T_{B, x}+V_{B} T_{B, y}\right\}, \\
& \mathcal{F}_{1}=\frac{\rho_{B} V_{B}}{\mu_{B}}+\frac{\kappa \chi}{R e}-\frac{\mu_{B, y}}{\mu_{B} R e}, \\
& \mathcal{F}_{2}=\chi\left\{\frac{\rho_{B} U_{B}}{\mu_{B}}-\frac{\chi \mu_{B, x}}{\mu_{B} R e}\right\}, \\
& \mathcal{F}_{3}=\frac{\mu_{B, y}}{\mu_{B}}, \\
& \mathcal{F}_{4}=\frac{\chi \mu_{B, x}}{\mu_{B}}, \\
& \mathcal{G}_{1}=r \chi^{2} U_{B, x x}+U_{B, y y}+s \chi V_{B, x y}-r \kappa \chi^{2} V_{B, x}, \\
& \mathcal{G}_{2}=\chi^{2} V_{B, x x}+r V_{B, y y}+s \chi U_{B, x y}+\kappa \chi^{2} U_{B, x}, \\
& \mathcal{G}_{3}=\chi^{2} W_{B, x x}+W_{B, y y}, \\
& \mathcal{G}_{4}=\chi^{2} T_{B, x x}+T_{B, y y}, \\
& \mathcal{G}_{5}=\Gamma\left(\chi^{2}\left(\kappa^{2} U_{B}^{2}+W_{B, x}^{2}\right)+r V_{B, y}^{2}+W_{B, y}^{2}+r \chi^{2}\left(U_{B, x}-\kappa V_{B}\right)^{2}+2 m \chi V_{B, y}\left(U_{B, x}-\kappa V_{B}\right)+\right. \\
& \left.2 \kappa \chi U_{B}\left(U_{B, y}+\chi V_{B, x}\right)+\left(U_{B, y}+\chi V_{B, x}\right)^{2}\right)-\kappa \chi T_{B, y}, \\
& \mathcal{H}_{1}=\frac{\chi\left(\Gamma P_{B, x}-\sigma \rho_{B} T_{B, x}\right)}{\mu_{B}}, \\
& \mathcal{H}_{2}=\frac{\Gamma P_{B, y}-\sigma \rho_{B} T_{B, y}}{\mu_{B}}, \\
& \mathcal{H}_{3}=\frac{\chi f_{T} T_{B, x}-\sigma \rho_{B} U_{B} R e}{\mu_{B}}, \\
& \mathcal{H}_{4}=\frac{f_{T} T_{B, y}-\sigma \rho_{B} V_{B} R e}{\mu_{B}}-\kappa \chi .
\end{aligned}
$$




\section{K-Operator}

The compressible $\mathbf{K}$-operator associated with the systems (B1) and (B2) is defined as

$$
\begin{aligned}
& K_{t}=\rho_{B}\left\{\mathbf{u} \cdot \mathbf{u}^{*}+\sigma T T^{*}\right\}+p\left\{\frac{p^{*}}{P_{B}}-\Gamma T^{*}\right\}-\frac{T p^{*}}{T_{B}} \\
& \frac{K_{x}}{\mu_{B}}=\frac{\chi \rho_{B} U_{B}}{\mu_{B}}\left\{\mathbf{u} \cdot \mathbf{u}^{*}+\sigma T T^{*}\right\}+\frac{\chi}{\mu_{B}}\left(u p^{*}+p u^{*}\right)+\frac{\chi U_{B}}{\mu_{B}}\left\{p\left(\frac{p^{*}}{P_{B}}-\Gamma T^{*}\right)-\frac{T p^{*}}{T_{B}}\right\}+\frac{\kappa \chi^{2}}{R e}\left\{e v u^{*}-2 u v^{*}\right\}- \\
& \frac{\chi \mathcal{F}_{4}}{\operatorname{Re}}\left\{\mathbf{u}_{\mathbf{1}} \cdot \mathbf{u}^{*}+T T^{*}\right\}+\frac{\chi \mathcal{F}_{3}}{\operatorname{Re}}\left(u v^{*}-v u^{*}\right)+\frac{s \chi}{R e}\left\{u\left(v_{y}^{*}-i b w^{*}\right)-u^{*}\left(v_{y}+i b w\right)\right\}+\frac{\chi^{2}}{R e}\left\{\mathbf{u}_{\mathbf{1}} \cdot u_{x}^{*}+T T_{x}^{*}-\left(\mathbf{u}_{\mathbf{1}} \cdot \mathbf{u}^{*}+T_{x} T^{*}\right)\right\}- \\
& \frac{\chi f_{T} T}{\mu_{B} R e}\left\{\mathcal{D}_{2} u^{*}+\mathcal{D}_{1} v^{*}+\chi W_{B, x} w^{*}+\chi T_{B, x} T^{*}\right\}-\frac{2 \chi \Gamma T^{*}}{R e}\left\{\mathcal{D}_{2} u+\mathcal{D}_{1} v+\chi W_{B, x} w\right\} \\
& \frac{K_{y}}{\mu_{B}}=\frac{\chi \rho_{B} V_{B}}{\mu_{B}}\left\{\mathbf{u} \cdot \mathbf{u}^{*}+\sigma T T^{*}\right\}+\frac{1}{\mu_{B}}\left(v p^{*}+p v^{*}\right)+\frac{V_{B}}{\mu_{B}}\left\{p\left(\frac{p^{*}}{P_{B}}-\Gamma T^{*}\right)-\frac{T p^{*}}{T_{B}}\right\}+\frac{\left(\kappa \chi-\mathcal{F}_{3}\right)}{R e}\left\{\mathbf{u}_{\mathbf{2}} \cdot \mathbf{u}^{*}+T T^{*}\right\}+ \\
& \frac{\mathcal{F}_{4}}{R e}\left(v u^{*}-u v^{*}\right)+\frac{1}{R e}\left\{\mathbf{u}_{\mathbf{2}} \cdot \mathbf{u}_{y}^{*}+T T_{y}^{*}-\left(\mathbf{u}_{2 y} \cdot \mathbf{u}^{*}+T_{y} T^{*}\right)\right\}+\frac{s}{R e}\left\{v\left(\chi u_{x}^{*}-i b w^{*}\right)-v^{*}\left(\chi u_{x}+i b w\right)\right\}- \\
& \frac{f_{T} T}{\mu_{B} R e}\left\{\mathcal{D}_{1} u^{*}+\mathcal{D}_{3} v^{*}+W_{B, y} w^{*}+T_{B, y} T^{*}\right\}-\frac{2 \Gamma T^{*}}{R e}\left\{\mathcal{D}_{1} u+\mathcal{D}_{3} v+W_{B, y} w\right\}, \\
& \frac{K_{z}}{\mu_{B}}=\frac{\chi \rho_{B} W_{B}}{\mu_{B}}\left\{\mathbf{u} \cdot \mathbf{u}^{*}+\sigma T T^{*}\right\}+\frac{1}{\mu_{B}}\left(w p^{*}+p w^{*}\right)+\frac{W_{B}}{\mu_{B}}\left\{p\left(\frac{p^{*}}{P_{B}}-\Gamma T^{*}\right)-\frac{T p^{*}}{T_{B}}\right\}+\frac{s \kappa \chi v w^{*}}{R e}+\frac{\mathcal{F}_{3}}{R e}\left(w v^{*}-v w^{*}\right)- \\
& \frac{\mathcal{F}_{4}}{R e}\left(w u^{*}+u w^{*}\right)-\frac{2 i b}{R e}\left\{\mathbf{u}_{\mathbf{3}} \cdot \mathbf{u}^{*}+T T^{*}\right\}+\frac{s}{R}\left\{w\left(\chi u_{x}^{*}+v_{y}^{*}\right)-w^{*}\left(\chi u_{x}+v_{y}\right)\right\}- \\
& \frac{f_{T} T}{\mu_{B} R e}\left\{\chi W_{B, x} u^{*}+W_{B, y} v^{*}+m \mathcal{D}_{4} w^{*}\right\}-\frac{2 \Gamma T^{*}}{R e}\left\{\chi W_{B, x} u+W_{B, y} v+m \mathcal{D}_{4} w\right\},
\end{aligned}
$$

where

$$
\begin{aligned}
& \mathbf{u}_{\mathbf{1}}=\{r u, v, w\}, \quad \mathbf{u}_{\mathbf{2}}=\{u, r v, w\}, \quad \mathbf{u}_{\mathbf{3}}=\{u, v, r w\} \\
& \mathbf{u} \cdot \mathbf{u}^{*}=u u^{*}+v v^{*}+w w^{*} \quad \text { and } \quad \mathbf{u}_{x}=\left\{u_{x}, v_{x}, w_{x}\right\}
\end{aligned}
$$

${ }^{1}$ W. S. Saric, H. L. Reed \& E. J. Kerschen, Boundary-layer receptivity to freestream disturbances, Annu. Rev. Fluid Mech. 34 (2002) 291-319.

${ }^{2}$ W. S. Saric, H. L. Reed \& E. B. White, Stability and transition of three-dimensional boundary layers, Annu. Rev. Fluid Mech. 35 (2003) 413-440.

${ }^{3}$ W. S. Saric, R. B. Carillo \& M. S. Reibert, Nonlinear stability and transition in 3-D boundary layers, Meccanica 33 (1998a) 469487

${ }^{4}$ H. Bippes, Basic experiments on transition in three-dimensional boundary layers dominated by crossflow instability, Prog. Aerosp. Sci. 35 (1999) 363412

${ }^{5}$ T. Herbert, Secondary instabilities of boundary layers, Ann. Rev. Fluid Mech. 20 (1988) 487-526.

${ }^{6}$ A. V. Federov, Excitation of waves of instability of the secondary flow in the boundary layer on a swept wing, J. Appl. Mech. Tech. Phys. 29 (1988) 643-648.

${ }^{7} \mathrm{M}$. Choudhari, Boundary-layer receptivity due to distributed surface imperfections of a deterministic or random nature, Theoret. Comput. Fluid Dyn. 4 (1993) 101-117.

${ }^{8}$ J. D. Crouch, Receptivity of three-dimensional boundary layers. AIAA, Aerospace Sciences Meeting and Exhibit, 31 st, Reno, NV. (1999)

${ }^{9} \mathrm{M}$. Choudhari, Roughness-induced generation of cross-flow vortices in three-dimensional boundary layers, Theoret. Comput. Fluid Dyn. 6 (1994) 1-30.

${ }^{10}$ S. S. Collis \& S. K. Lele, Receptivity to surface roughness near a swept leading edge, J. Fluid Mech. 380 (1999) $141-168$.

${ }^{11} \mathrm{P}$. Wassermann \& M. Kloker, Mechanisms and passive control of crossflow-vortex-induced transition in a three-dimensional boundary layer, J. Fluid Mech. 456 (2002) 49-84.

${ }^{12}$ L. U. Schrader, L. Brandt \& D. S. Henningson, Receptivity mechanisms in three-dimensional boundary layer flows, J. Fluid Mech. 618 (2009) 209-241. 
${ }^{13}$ L. U. Schrader, S. Amin \& L. Brandt, Transition to turbulence in the boundary layer over a smooth and rough swept plate exposed to free-stream turbulence, J. Fluid Mech. 646 (2010) 297-325.

${ }^{14}$ M. S. Reibert, W. S. Saric, R. B. Carillo \& K. L. Chapman, Experiments in nonlinear saturation of stationary crossflow vortices in a swept-wing boundary layer. AIAA Paper 96-0184 (1996)

${ }^{15}$ W. S. Saric, R. B. Carillo \& M. S. Reibert, Leading-edge roughness as a transition control mechanism, AIAA Paper 98-0781 (1998b)

${ }^{16}$ R. H. Radeztsky, M. S. Reibert \& W. S. Saric, Effect of isolated micron-sized roughness on transition in swept-wing flows. AIAA J. 37 (1999) 1370-1377.

${ }^{17}$ L. L. Ng \& J. D. Crouch, Roughness-induced receptivity to crossflow vortices on a swept wing, Phys. Fluids 11 (1999) 432.

${ }^{18}$ D. Tempelmann, L.- U. Schrader, A. Hanifi, L. Brandt \& D. S. Henningson, Swept wing boundary-layer receptivity to localized surface roughness, J. Fluid Mech. 711 (2012) 516-544

${ }^{19}$ S. M. Mughal \& R. Ashworth, Uncertainty quantification based receptivity modelling of crossflow instabilities induced by distributed surface roughness in swept wing boundary layers. AIAA Paper 2013-3106 (2013)

${ }^{20}$ S. M. Mughal, Advanced Transition Prediction and Development of Linearised Navier-Stokes Receptivity Methods, Validation and Application. Institute for Mathematical Sciences, Imperial College London, EADS-IW ref: IW102175 (2012).

${ }^{21}$ H. Salwen \& C. E. Grosch, The continuous spectrum of the Orr-Sommerfeld equation. Part 2. Eigenfunction equations, J. Fluid Mech. 104 (1981) 445-465.

${ }^{22}$ D. C. Hill, Adjoint systems and their role in the receptivity problem for boundary layers, J. Fluid Mech. 272 (1995) $183-204$.

${ }^{23}$ C. Airiau, Non-parallel acoustic receptivity of a Blasius boundary layer using an adjoint approach, Flow, Turbulence and Combustion 65 (2000) 347-367.

${ }^{24}$ A. Dobrinsky \& S. S. Collis, Adjoint parabolized stability equations for receptivity prediction. AIAA Paper (2000) $2000-2651$.

${ }^{25}$ A. Dobrinsky, Adjoint analysis for receptivity prediction. PhD Thesis, Rice University. (2003)

${ }^{26}$ J.O. Pralits, C. Airiau, A. Hanifi, D.S. Henningson, Sensitivity Analysis Using Adjoint Parabolized Stability Equations for Compressible Flows, Flow, Turbulence and Combustion, 65 (2000) 321-346

${ }^{27}$ P. Luchini \& A. Bottaro, Gortler vortices: a backward-in-time approach to the receptivity problem, J. Fluid Mech. 363 (1998) 1-23.

${ }^{28}$ J. O. Pralits, A. Hanifi \& D. S. Henningson, Adjoint-based optimisation of steady suction for disturbance control in incompressible flows, J. Fluid Mech. 467 (2002) 129-161.

${ }^{29}$ F. Giannetti \& P. Luchini, Leading-edge receptivity by adjoint methods, J. Fluid Mech. 547 (2006) 21-53.

${ }^{30}$ N. K. Yamaleev, B. Diskin \& E. J. Nielsen, Adjoint-based methodology for time-dependent optimization, AIAA-2008-5857, 12th AIAA/ISSMO Multidisciplinary Analysis and Optimization Conference, Victoria, BC, Canada.

${ }^{31}$ E. J. Nielsen, B. Diskin \& N. K. Yamaleev, Discrete adjoint-based design of optimization of unsteady turbulent flows on dynamic unstructured grids, AIAA J. 48 (2010) 1195-1206.

${ }^{32}$ D. Tempelmann, A. Hanifi \& D. S. Henningson, Swept-wing boundary layer receptivity, J. Fluid Mech. 700 (2012) $490-501$.

${ }^{33}$ M. H. Carpenter, M. Choudhari, F. Li, C. L. Streett \& C.-L. Chang, Excitation of crossflow instabilities in a swept wing boundary layer, AIAA Paper, (2010) 2010-378.

${ }^{34}$ P. Luchini \& A. Bottaro, Adjoint equations in stability analysis, Annu. Rev. Fluid Mech. 46 (2014) 493517

${ }^{35}$ P. R. Ashill, C. J. Beth \& M. Gaudet, A wind tunnel study of transitional flows on a swept panel wing at high subsonic speeds, CEAS 2nd European forum on laminar flow technology, (1996).

${ }^{36}$ Technical documentation of the DLR TAU-code release 2011.2.0. Institute of Aerodynamics and Flow Technology, Braunshweig.

${ }^{37}$ C. Thomas, S. M. Mughal, M. Gipon, R. Ashworth \& A. Martinez-Cava, Stability of an Infinite Swept Wing Boundary Layer with Surface Waviness, AIAA J. 50(10) (2016) 3024-3038.

${ }^{38}$ J. R. Edwards \& S. Chandra, Comparison of Eddy-Viscosity Transport Turbulence Models for Three-Dimensional, Shock Separated Flowfields, AIAA J. 34 No. 4 (1996) 756-763.

${ }^{39}$ S. M. Mughal, Transition prediction in fully 3D compressible flows, Imperial College London, Final report prepared for QinetiQ and British Aerospace (MAD) (2001).

${ }^{40}$ H.B. Keller \& T. Cebeci, Accurate Numerical Methods for Boundary Layer Flows II: Two Dimensional Turbulent Flows, AIAA J. 10 (1972) 1193-1199.

${ }^{41}$ S. M. Mughal, Active Control of Wave Instabilities in Three-Dimensional Compressible Flows, Theoret. Comput. Fluid Dyn. 12 (1998) 195-217.

${ }^{42}$ C. Davies \& P. W. Carpenter A novel velocity-vorticity formulation of the Navier-Stokes equations with applications to boundary layer disturbance evolution, J. Comp. Phys. 172 (2001) 119165. 